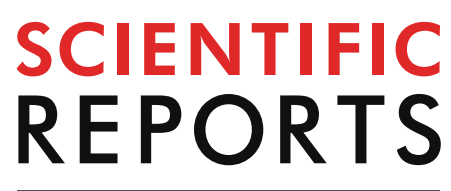

natureresearch

Check for updates

\title{
Genome wide in-silico miRNA and target network prediction from stress responsive Horsegram (Macrotyloma uniflorum) accessions
}

\author{
Jeshima Khan Yasin ${ }^{1,11 凹}$, Bharat Kumar Mishra ${ }^{1,10,11}$, M. Arumugam Pillai ${ }^{2}$, \\ Nidhi Verma ${ }^{3}$, Shabir H. Wani ${ }^{4}$, Hosam O. Elansary5,6, Diaa O. El-Ansary ${ }^{7}$, P. S. Pandey ${ }^{8}$ \& \\ Viswanathan Chinnusamy ${ }^{9}$
}

Horsegram (Macrotyloma uniflorum (Lam.) Verdc.) is a drought hardy food and fodder legume of Indo-African continents with diverse germplasm sources demonstrating alternating mechanisms depicting contrasting adaptations to different climatic zones. Tissue specific expression of genes contributes substantially to location specific adaptations. Regulatory networks of such adaptive genes are elucidated for downstream translational research. MicroRNAs are small endogenous regulatory RNAs which alters the gene expression profiles at a particular time and type of tissue. Identification of such small regulatory RNAs in low moisture stress hardy crops can help in cross species transfer and validation confirming stress tolerance ability. This study outlined prediction of conserved miRNAs from transcriptome shotgun assembled sequences and EST sequences of horsegram. We could validate eight out of 15 of the identified miRNAs to demonstrate their role in deficit moisture stress tolerance mechanism of horsegram variety Paiyur1 with their target networks. The putative mumiRs were related to other food legumes indicating the presence of gene regulatory networks. Differential miRNA expression among drought specific tissues indicted the probable energy conservation mechanism. Targets were identified for functional characterization and regulatory network was constructed to find out the probable pathways of post-transcriptional regulation. The functional network revealed mechanism of biotic and abiotic stress tolerance, energy conservation and photoperiod responsiveness.

MicroRNAs (miRNAs/ miRs) are 19-24 nucleotides long endogenous molecular bigwigs in post-transcriptional gene regulatory networks ${ }^{1}$. These $m i R$ genes are capped, polyadenylated and spliced like other RNA polymerase II transcripts. The mature miRs are located in a hairpin structure within the primary transcript (pri-transcript) and are preprocessed by at least two RNase mediated steps to mature miRNA. Pri-miRNAs range from 50-100 nucleotides that coil into hairpin loop structures encompassing paired stems and unpaired loops ${ }^{2}$. Intensive

\footnotetext{
${ }^{1}$ Division of Genomic Resources, ICAR-National Bureau Plant Genetic Resources, PUSA Campus, New Delhi 110012, India. '2Department of Plant Breeding and Genetics, Agricultural College and Research Institute, Tamil Nadu Agricultural University, Killikulam, Vallanadu, Tamil Nadu 628252, India. ${ }^{3}$ Principal Scientist (Education Planning and Home Science), Agricultural Education Division Krishi Anusandhan Bhawan I, Indian Council of Agricultural Research, PUSA Campus, New Delhi 110 012, India. ${ }^{4}$ Mountain Research Centre For Field Crops, Khudwani Anantnag-192101, Sher-E-KashmiR University of Agricultural Sciences and Technology of Kashmir, Badgam, J\&K, India. ${ }^{5}$ Plant Production Department, College of Food and Agricultural Sciences, King Saud University, P.O. Box 2455, Riyadh 11451, Saudi Arabia. ${ }^{6}$ Floriculture, Ornamental Horticulture, and Garden Design Department, Faculty of Agriculture (El-Shatby), Alexandria University, Alexandria 21545, Egypt. ${ }^{7}$ Precision Agriculture Laboratory, Department of Pomology, Faculty of Agriculture (El-Shatby), Alexandria University, Alexandria, Egypt. ${ }^{8}$ Indian Council of Agricultural Research (ICAR), PUSA, New Delhi 110 012, India. ${ }^{9}$ Division of Plant Physiology, Indian Agricultural Research Institute, New Delhi 110012, India. ${ }^{10}$ Present address: Department of Biology, University of Alabama at Birmingham, Birmingham, AL 35294-1170, USA. ${ }^{11}$ These authors contributed equally: Jeshima Khan Yasin and Bharat Kumar Mishra. ${ }^{\square}$ email:Yasin.Jeshima@icar.gov.in
} 
investigation over decades has enriched the miRNA's knowhow in biogenesis and explicit regulatory machinery. Plant miRNA precursors are less conserved, whereas mature miRNAs are conserved at higher magnitude in comparison to animal miRNAs ${ }^{3}$. Hence, exploring conserved miRNAs makes sense to identify the potential miRNAs from flora with targeted traits.

Macrotyloma uniflorum (Lam.) Verdc. (Horsegram) originated from South-Western India is a multipurpose pulse crop mainly cultivated for providing nutritional security in the form of food, livestock supplement and green manure in India and Africa ${ }^{4-6}$. Being a diploid $(2 n=20,22$ or 24], short duration (120-180 days to maturity) plant species and adapted to grow on wide range of agro-climatic conditions, horsegram can be weighed as appropriate model for moisture stress tolerance genes/QTLs investigation challenging undernourishment in drought prone regions $s^{6,7}$. Further, it can be envisaged as nutraceuticals, forage crop ${ }^{8}$ and anti-calcifying inhibitors ${ }^{9}$. Recently, substantial improvement in accumulation of EST ${ }^{5,10}$ and transcriptome data ${ }^{11}$ has compelled its position as a future crop with versatile utility. The present study details the comprehensive computational approach $^{12-14}$ to predict miRNAs fromavailable ESTs and Transcriptome Shotgun Assembly (TSA) sequences of horsegram based on miRNA homolog search. Potential pathways contributing to drought tolerance is studied as inherent trait of horsegram, which could have common or divergent gene regulatory networks $\mathrm{s}^{6,10,15}$.

Flora without whole genome sequences are having alternate resource sequence sources in public databases such as Genome Survey Sequences (GSS), Expressed Sequence Tags (EST) and Bacterial Artificial Chromosome (BAC) sequences rendering plentiful resources to mine conserved microRNAs ${ }^{16-18}$. The database miRBase hosts 8746 reported miRNAs belonging to four phyla of plant species enlisted both mature and precursor miRNAs (https://www.miRbase.org/) (Release 21, June 2014). Plant miRs share functional similarity with small interfering RNAs (siRNAs) in guided target cleavage as microRNA targets sites of coding $m R N A$ sequences ${ }^{3,19}$. Currently, the data generated from next generation sequencing (NGS) studies have been employed in miRNAs prediction and their impact on multiple traits in related species. The in-silico homology based prediction of miRNA is advantageous and orthologs of previously reported miRNAs could be deciphered with their evolutionary significance among species ${ }^{20-22}$. In recent years, the multi-faceted functionalities of $m i R s$ in plants are being understood effectively despite the fact that $m i R N A$ s are less studied in plants.

This is the first report in horsegram with comprehensive analysis of conservation and phylogeny of miRs. Previously, a comprehensive study was performed in horsegram from ESTs in predicting eight novel $m i R s^{11}$. Here we report differential expression of identified miRs through a stringent in-silico schema elucidating conserved miRNAs, their characterization, validation with their target annotation and networking.

\section{Materials and methods}

Query and reference datasets. Totalling 27,997 shotgun assembled contigs of transcriptome were collected from TSA sequence set: GANR01000001-GANR01027997 from European Nucleotide Archive (www.ebi. ac.uk/ena), SSH-Mu library of moisture stressed cDNA of Macrotyloma (https://www.ncbi.nlm.nih.gov/nucest NCBI dbEST ID 75866463 from) and 1008 ESTs were downloaded (www.ncbi.nlm.nih.gov/dbEST/) from publically available NCBI EST database to represent query sequences for miRNA homolog search. About $8496 \mathrm{miR}$ Base mature Viridiplantae miRNAs were used (https://www.miRbase.org) (Released 21: June, 2014) database ${ }^{23}$ in the present investigation and clustered by CD- HIT-EST, with threshold value of $100^{24}$. Out of 3777 clustered sequences, only non-redundant miRNAs were used as reference miRNAs for finding the homologs in M. uniflorum (Lam.) Verdc. candidate sequences to create a local nucleotide sequence database. To elucidate likely role of miRNAs involved in drought stress tolerance trait, the predicted microRNAs were screened for target genes listed in DroughtdB database (https://pgsb.helmholtz-muenchen.de/droughtdb/).

Bioinformatics tools employed. For conserved miRNA prediction of horsegram candidate sequences from Viridiplantae miRNAs reported in miRBase database, NCBI BLAST version 2.2.27 25 an alignment tool was used (https://ftp.ncbi.nih.gov/blast/executables/blast+). The representative sequences of all plant miRNAs were obtained after clustering with CD-HIT-EST with threshold value of $100^{24}$. MFOLD online tool ${ }^{26}$ was (https://

\begin{tabular}{|l|l|l|l|l|l|l|l|l|l|l|l|l|l|}
\hline TSA ID & MFP & LT & LT & LP & AU\% & GC\% & A & C & G & U/T & MFE & AMFE & FEI \\
\hline GANR01006328 & $m i R 482$ & 355 & 355 & 119 & 57.99 & 42.01 & 38 & 28 & 22 & 31 & -42.01 & -31.34 & 0.74 \\
\hline GANR01007318 & $m i R 482$ & 460 & 460 & 130 & 39.24 & 40.76 & 38 & 21 & 32 & 39 & -40.76 & -35.61 & 0.87 \\
\hline GANR01008465 & $m i R 156$ & 609 & 609 & 100 & 50 & 50 & 31 & 19 & 19 & 31 & -50 & -39 & 0.78 \\
\hline GANR01009673 & $m i R 2673$ & 775 & 775 & 118 & 53.39 & 46.61 & 28 & 17 & 38 & 35 & -46.61 & -26.77 & 0.57 \\
\hline GANR01016903 & $m i R 5653$ & 2143 & 2143 & 119 & 52.11 & 47.89 & 36 & 25 & 32 & 26 & -47.89 & -28.49 & 0.59 \\
\hline GANR01019897 & $m i R 156$ & 670 & 670 & 107 & 56.08 & 43.92 & 30 & 26 & 21 & 30 & -43.92 & -26.82 & 0.61 \\
\hline GANR01022354 & $m i R 1507$ & 950 & 950 & 91 & 54.45 & 45.05 & 31 & 23 & 18 & 19 & -45.05 & -36.04 & 0.8 \\
\hline GANR01022920 & $m i R 168$ & 515 & 515 & 122 & 41.81 & 58.19 & 19 & 36 & 35 & 32 & -58.19 & -48.85 & 0.83 \\
\hline GANR01023649 & $m i R 2118, m i R 482$ & 644 & 644 & 106 & 60.38 & 39.62 & 36 & 19 & 23 & 28 & -39.62 & 29.05 & 0.7 \\
\hline GANR01024080 & $m i R 390$ & 576 & 576 & 102 & 56.87 & 43.13 & 24 & 24 & 20 & 29 & -43.13 & 44.41 & 1.03 \\
\hline
\end{tabular}

Table 1. Characteristics of predicted precursor miRNAs of horsegram. MFP, microRNA family/families present in stem-loop structure; LT, length of TSA; LP, length of precursor miRNA; MFE -minimal folding free energy (-Kcal/mol); AMFE, adjusted minimal folding (-Kcal/mol); MFEI, minimal folding free energy index. 


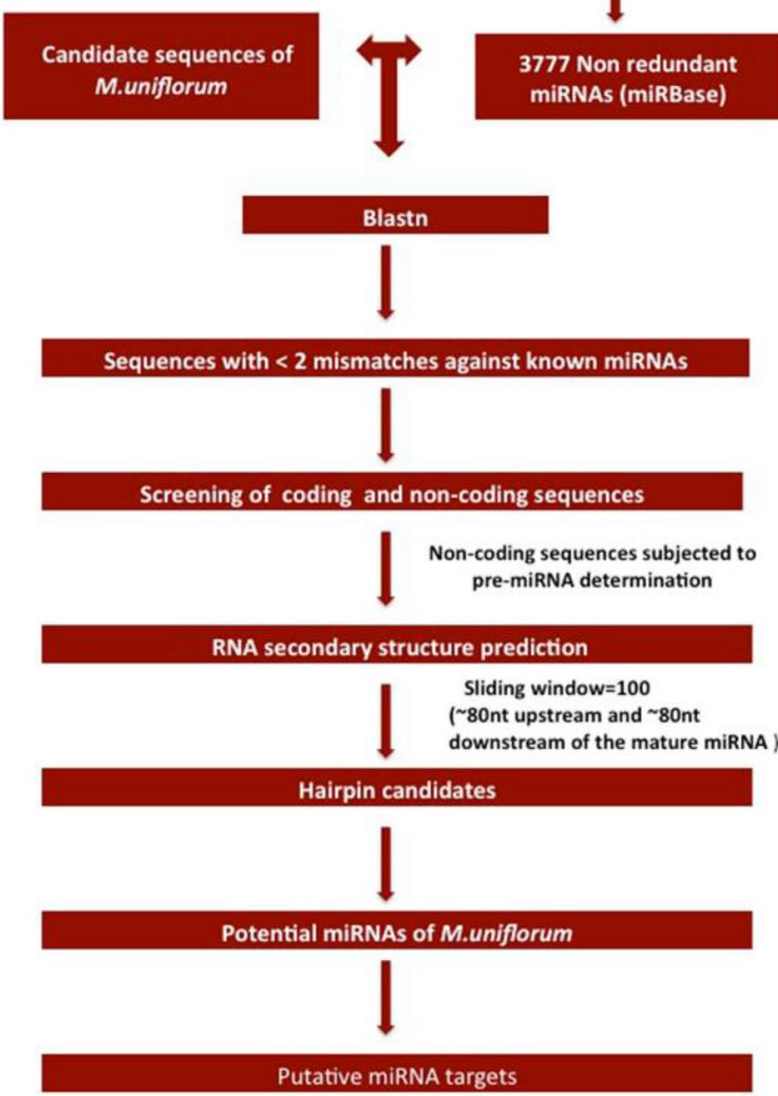

Figure 1. Pipeline for prediction of miRNAs and its in-silico validation. From non-coding seqeuences and secondary structure analyses miRs were predicted in-silico as depicted in this pipeline.

unafold.rna.albany.edu/?q=mfold) used for pre-miRNAs secondary structures prediction. The miRNA targets were deduced using psRNAtarget server ${ }^{27}$. Online circoletto ${ }^{28}$ tool was used to illustrate the miRNAs and its target genes by circos $\operatorname{plot}^{29}$. The phylogenetic analysis of predicted miRNAs with their closely related miRNA families was performed with MEGA $7^{30}$.

Computational prediction of potential miRNA homologs. The prediction of miRNA homologs was performed using TSA and EST sequences of M. uniflorum available at NCBI and EBI databases. The plant miRNAs were obtained from NCBI was clustered and the representative sequences generated were employed as reference miRNA database using BLAST-2.2.27+. The query sequence consisting of TSA and EST of horsegram were subjected to nucleotide blast (blastn) against reference sequences of plant miRNAs with the following set parameters: (i) word match size-7; (ii) length of mature $m i R N A$ sequence $\geq 18$ nt without gap; (iii) mismatch range- $0-2$ (iv) e-value-0.1. The filtered sequences were utilized to find out the coding and non-coding candidate sequences by performing blastx against non-redundant protein database. The protein coding regions were removed whereas the non-coding regions were exploited for secondary structure prediction and validation.

Secondary structure prediction and validation. With maximum stringency only non-coding sequences were subjected to Zuker's folding algorithm based secondary structure prediction in MFold ${ }^{26}$. A sliding window of $100 \mathrm{nt}$ from $\sim 80 \mathrm{nt}$ upstream and $\sim 80 \mathrm{nt}$ nucleotide downstream of the mature miRNA were set to find the precursors ${ }^{33}$ with parameters set as: (a) Folding temperature- $37^{\circ} \mathrm{C}$, (b) Ionic conditions of $1 \mathrm{M} \mathrm{NaCl}$ without divalent ions, (c) linear RNA sequence, (d) percent sub-optimality number of 5 and (e) maximum interior/bulge loop size-30. As reported elsewhere, to validate the structures of pre-miRs adjusted minimal folding free energy (AMFE) and minimal folding free energy index (MFEI) were calculated ${ }^{34}$ (Table 1).

The secondary structures were screened for ${ }^{35}$ : (i) Less than three nucleotide substitutions within predicted mature $m i R N A$ to reference $m i R$, (ii) The candidate sequence must fold into an appropriate and proper stemloop hairpin secondary structure, (iii) The localization of mature miRNA must be in one arm of the stem-loop structure, (iv) Less than six mismatches between mature miRNA and its corresponding star sequence $\left(m i R N A^{*}\right)$ 


\begin{tabular}{|l|l|l|l|l|l|l|}
\hline Horsegram $m i R N A$ & miRNA homolog & Mature miRNA sequence & LM & NM & Loc & Strand \\
\hline mun-miR482d-3p & gma-miR482d-3p & gguaugggagguguagggaaga & 22 & 0 & $3^{\prime}$ & - \\
\hline mun-miR482b-5p & gma-miR482b-5p & uuccuucccaauccccccaua & 21 & 0 & $5^{\prime}$ & - \\
\hline mun-miR482a-5p & gma-miR482a-5p & agaauuugugggaaugggcuga & 22 & 0 & $5^{\prime}$ & + \\
\hline mun-miR482-5p & pvu-miR482-5p & ggaaugggcugauugggaagca & 22 & 0 & $5^{\prime}$ & + \\
\hline mun-miR482a-3p & gma-miR482a-3p & ucuucccaauuccgcccauuccua & 24 & 0 & $3^{\prime}$ & + \\
\hline mun-miR156r & gma-miR156r & ugcucucuaucuucugucag & 20 & 0 & $5^{\prime}$ & - \\
\hline mun-miR2673a & mtr-miR2673a & ucuuccucuuccucuucc & 18 & 0 & $3^{\prime}$ & + \\
\hline mun-miR5653 & ath-miR5653 & ugaguugaguugaguugag & 19 & 1 & $3^{\prime}$ & + \\
\hline mun-miR156k & osa-miR156k & gacagaagagagagagcaca & 20 & 0 & $5^{\prime}$ & + \\
\hline mun-miR1507a & gma- $m i R 1507 \mathrm{a}$ & agacgauguauggaaugaga & 20 & 0 & $3^{\prime}$ & - \\
\hline mun-miR168a-5p & ath-miR168a-5p & ucgcuuggugcaggucgggaa & 21 & 0 & $5^{\prime}$ & + \\
\hline mun-miR2118 & pvu-miR2118 & aggaauggguggaaucggcaa & 21 & 0 & $3^{\prime}$ & - \\
\hline mun-miR482a & sly-miR482a & aggaauggguggaauuggaaa & 21 & 2 & $3^{\prime}$ & - \\
\hline mun-miR390b-5p & gma-miR390b-5p & gugcuaucccuccugagcuu & 20 & 0 & $5^{\prime}$ & - \\
\hline mun-miR390a-5p & ath- $m i R 390 \mathrm{a}-5 \mathrm{p}$ & gcgcuaucccuccugagcuu & 20 & 1 & $5^{\prime}$ & - \\
\hline
\end{tabular}

Table 2. Details of the predicted horsegram miRNAs (tentative names given to putative predicted miRNAs. Submitted to MiRBase). LM, Length of the mature miRNA.
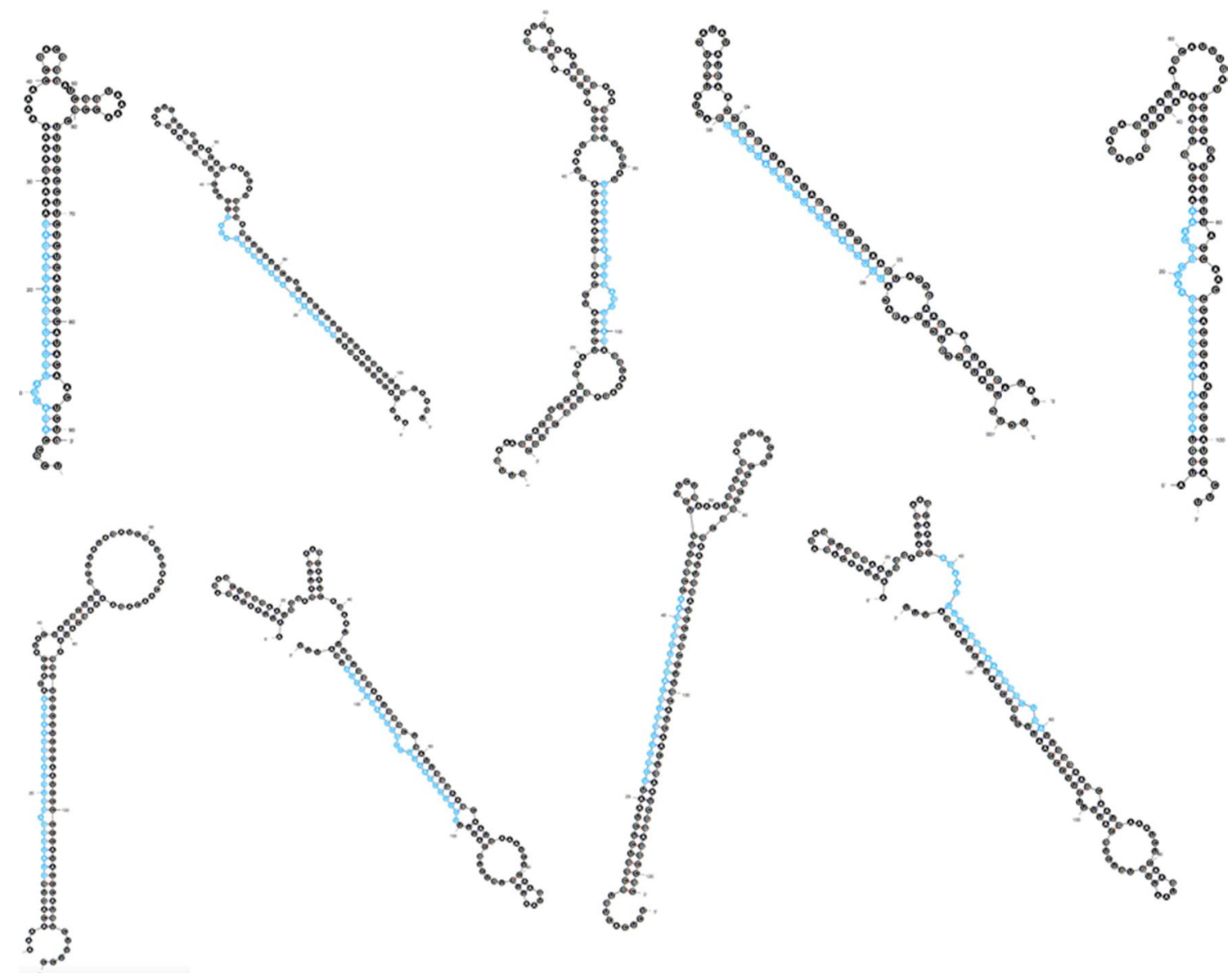

Figure 2. Secondary structures of predicted miRNA precursors of horsegram using MFOLD tool ${ }^{26}$. 


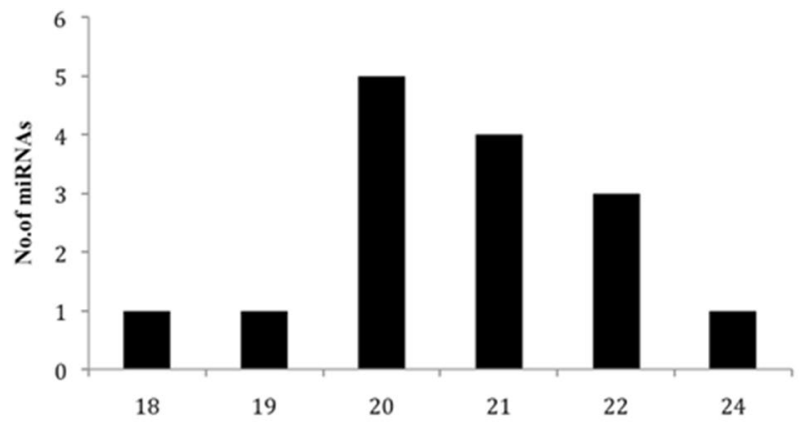

a

Length of potential miRNAs

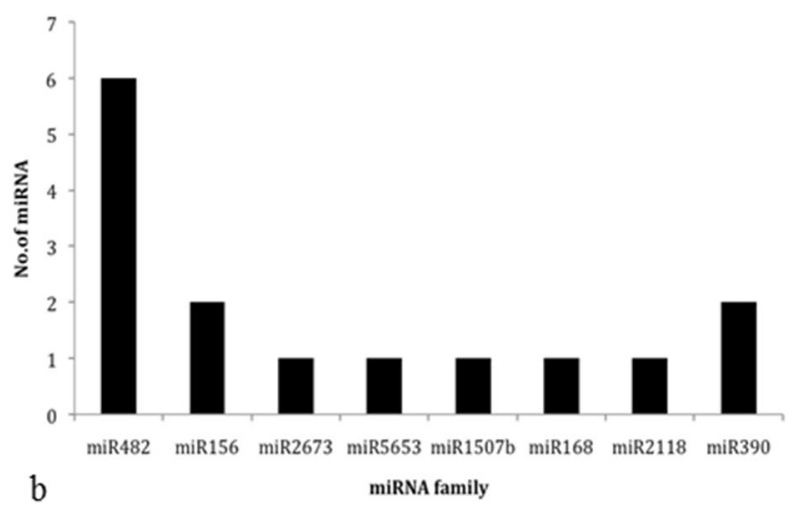

Figure 3. Identified $m i R N A$ s with (a) length variation and (b) family size in horsegram. The differentially expressed miRs were found to have more members in horsegram indicating network of $n c R N A$ s playing key role in regulating stress tolerance mechanism.

\begin{tabular}{|l|l|l|l|l|}
\hline Parameter & Mean & Standard Deviation & Minimum & Maximum \\
\hline LP & 111.4 & 12.02 & 91 & 130 \\
\hline LM & 20.733 & 1.437 & 18 & 24 \\
\hline AU\% & 52.232 & 6.86 & 39.24 & 60.38 \\
\hline GC\% & 45.718 & 25.82 & 39.62 & 58.19 \\
\hline A & 31.6 & & 19 & 38 \\
\hline U/T & 32.31 & & 58.1 & 19 \\
\hline G & 26 & & 18 & 38 \\
\hline C & 23.8 & & 17 & 36 \\
\hline MFE & -31.06 & & -37.3 & -59.6 \\
\hline AMFE & 28.37 & & -31.34 & -48.85 \\
\hline MFEI & 0.752 & & 0.57 & 1.03 \\
\hline
\end{tabular}

Table 3. Summary statistics for precursor miRNAs of M. uniflorum. LP, Length of the pre-cursor miRNA; LM, Length of the mature miRNA.

and (v) The secondary structure of putative pre-miR must contain high negative MFE and MFEI levels. Putative horsegram miRNAs were designated based on the standard nomenclature system ${ }^{23,36}$.

Phylogeny of horsegram miRNA. To enumerate the conserved nature and its phylogenetic relationship of $m i R s$ and its precursors were aligned with reported plant miRNAs using BLASTn with e-value, maximum mismatch and hits number set as 10, 3 and 5 respectively. The homologous precursor miRNAs were aligned to predicted miRNAs with Phylogeny.fr web tool (https://www.phylogeny.fr/index.cgi) ${ }^{37}$ by integrating multiple sequence alignment tools, MUSCLE ${ }^{38}$ and GBlocks ${ }^{39}$ to refine the alignment; phylogenetic tree construction by PhyML ${ }^{40}$ and Tree rendering by TreeDyn ${ }^{41}$. The alignment output is used in MEGA $7^{30}$ for interpretation of molecular clock to estimate interfamily evolution. 


\begin{tabular}{|l|l|}
\hline Database accessed & $\begin{array}{l}\text { NCBI dbEST } \\
\text { EBI }\end{array}$ \\
\hline Candidate number of ESTs & 1050 \\
\hline Candidate number of TSA & 27,997 \\
\hline Total number of candidate sequences & 29,047 \\
\hline Candidate number of miRNAs & 8496 \\
\hline Number of contigs containing potential precursors & 10 \\
\hline Number of microRNA families & 8 \\
\hline Number of microRNAs predicted & 15 \\
\hline Frequency of horsegram miRNA & 1 miRNA per 1937(approx) sequences \\
\hline
\end{tabular}

Table 4. Summary of the outcomes of the bioinformatics approach adopted to identify miRNAs in horsegram.

Phylogenetic analysis. The analysis involved 146 nucleotide sequences. All positions containing gaps and missing data were eliminated. Evolutionary analyses were also conducted in MEGA7 ${ }^{30}$.

Functional annotation of miRNA targets. As annotations are not available for horsegram, the closely related soybean was used as a reference to annotate $m i R$ targets of the predicted miRNAs. Putative identified query miRs were hit against horsegram $m R N A$ sequences using psRNATarget tool ${ }^{27}$. The parameters set target prediction were (i) 9-11 nucleotide mismatch range for translational inhibition, (ii) maximum 4 mismatches without gap at complementary site, (iii) multiplicity of target sites -2 , (iv) maximum expectation value -3 and (v) number of hits -10 . The homologs of putative miRNAs and their targets were represented in Glycine max genome as circos plot to demonstrate multiple targets of identified putative miRNAs in horsegram ${ }^{28}$. In addition, to elucidate the role of identified miRs in drought stress response, specific miRNA target genes among drought tolerance contributing genes reported in Droughtdb (https://pgsb.helmholtz-muenchen.de/droughtdb/) were confirmed. The genes reported for horsegram are insufficient, thus, $A$. thaliana was used as reference organism to determine gene targets for the new miRNAs predicted. The identified putative miRNAs were used as query against the $A$. thaliana DFCI gene index (AGI) release 15 and A. thaliana TAIR10, cDNA, removed miRNA gene (release date 14th December 2010) using psRNATarget tool. The parameters set for the target gene prediction were as follows: (i) range of central mismatch for translational inhibition 9-11 nucleotide; (ii) a maximum of 64 mismatches without gaps at complementary site; (iii) multiplicity of target sites 2; (iv) maximum expectation value 3 and (v) Number of hits -5 . Similarly, to understand the specific possibilities of putative horsegram miRNAs involved in drought stress, the drought genes were downloaded from Droughtdb in order to search for drought miRNA target genes. The visual representation of $m i R N A$ and its targets was represented by drawing circus plot to show the multiple targets of horsegram microRNAs predicted.

Functional annotation and metabolic pathway analysis. Mercator ${ }^{42}$ AgriGO $^{43}$ and $\mathrm{B}^{2} \mathrm{~g}^{44}$ were used to determine the functional roles and to classify Gene Ontology (GO) terms into molecular functions, biological processes and cellular components. Further, corresponding pathways were mined through KASS server, which assigns KEGG Orthology (KO) terms and employs KEGG pathways for miRNA target genes. The simplified schema of workflow adopted in present investigation for miRNA prediction is given in Fig. 1 .

Quantitative expression analysis of identified miRNAs. Stress responsive horsegram germplasm were identified from the germplasm core developed as described earlier (6). Selected stress responsive variety Paiyur 1 was raised in glass house under control and moisture stress conditions. Plants were maintained at field condition for control and at temporary wilting point for stress condition. Leaf tissues of control and stress plants were collected and fixed in RNALater and stored at $-80^{\circ} \mathrm{C}$ till RNA isolation.

High quality RNA samples were extracted from $100 \mathrm{mg}$ (wet weight) leaf tissue using Plant RNA Easy mini spin coloumn kit following the manufacturer's guidelines (Qiagen). Quantity and quality of the RNA was quantified with the advanced Qiaexpert.

RNA samples of one microgram each were 3' polyadenylated using Poly A RNA polymerase (Sigma Aldrich). Forward primers sequences of all $15 \mathrm{miRs}$ and a common Poly T reverse primers were designed (Supplementary material 1).

Quantitative rtPCR using One Step PrimeScript ${ }^{\text {tix }}$ RT-PCR Kit with SYBR green (Takara) was performed for all 15 identified miRs. qPCR capturing was conducted using the Illumina Eco RT PCR machine.

For qPCR cycling conditions set were $50^{\circ} \mathrm{C}$ for $10 \mathrm{~min}$ for cDNA sysnthesis; $95^{\circ} \mathrm{C}$ for 3 min for polymerase activation followed by $45^{\circ}$ cycles of $95^{\circ} \mathrm{C} 15 \mathrm{~s}, 60^{\circ} \mathrm{C}$ for $30 \mathrm{~s}$ capturing with SYBR green filter at end of each cycle.

\section{Results}

miRNAs in M.uniflorum. Significant miRNA homologs within reported 8496 miRNAs were identified by executing nucleotide blast (BLASTn) with 27,997 TSA contigs, SSH-Mu library sequences of moisture stressed horsegram cDNA (NCBI dbEST ID 75866463) and 1008 EST sequences as query resulted in 16 ESTs and 6303 TSA hits (E value 0.1 ) for further analysis. Stringent filtering (mismatch $<3$ and length $>17$ ) was incorporated to narrow down the hits to 5807 (8 ESTs and 5799 TSA) sequences for putative candidate sequences identifica- 


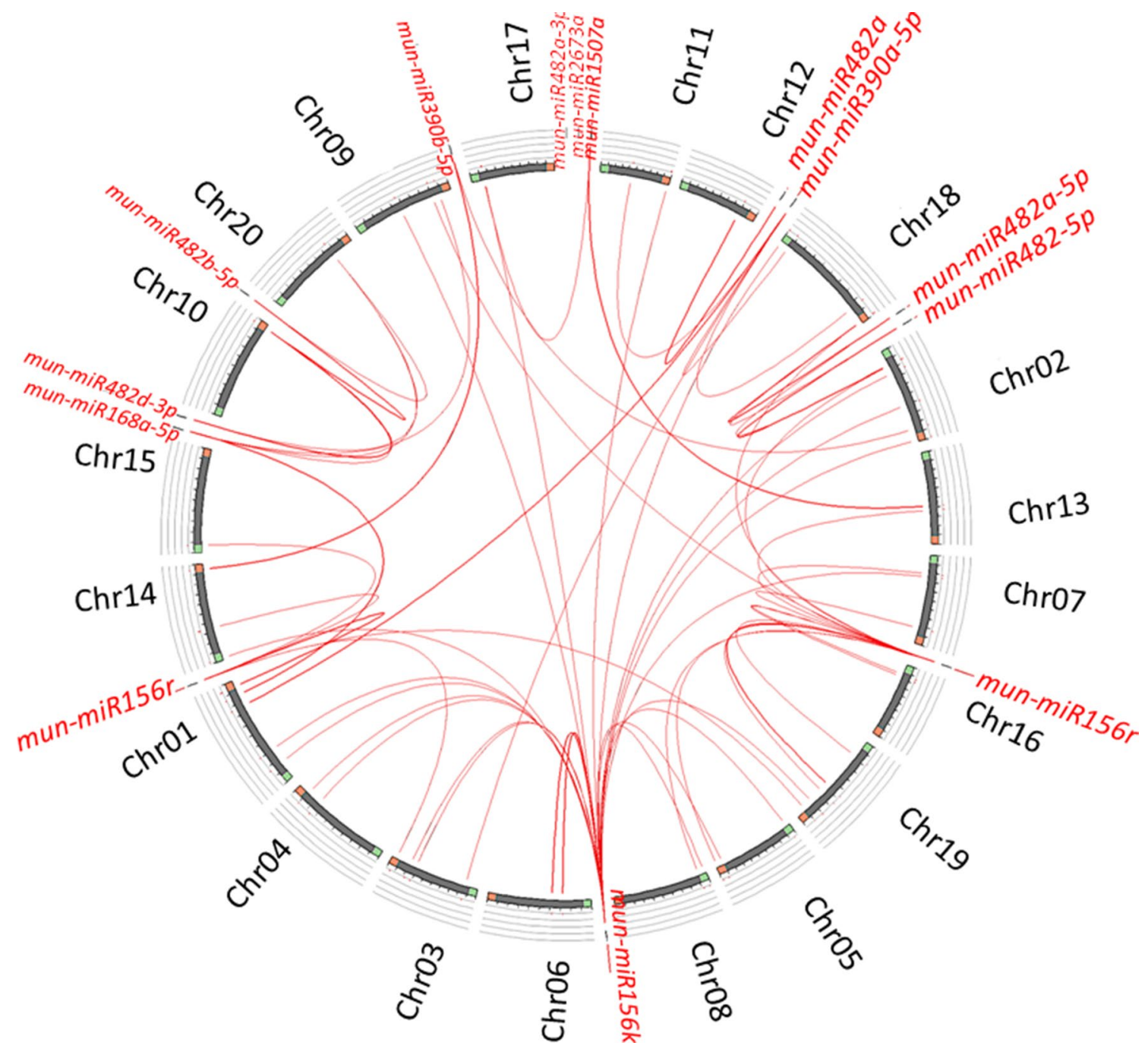

Figure 4. Synteny mapping of putative horsegram $m i R s$ with soybean genome. Synteny map explains the conserved sequences across species and their cross species transferability.

tion with non-coding regions. Based on coding potential, 214 noncoding sequences (7 ESTs and 207 TSA) were filtered out to predict ten distinct pre-miRs coding for 15 conserved mature $m i R s$ (Tables 1 and 2) clustering into nine different $m i R$-families (Figs. 2 and 3 ).

Secondary structure prediction and validation. The predicted miRNAs were analyzed for various structural features to distinguish from other small RNAs such as tRNAs, $r R N A$ s and $m R N A$ s (Table 1 and Fig. 2). Most crucial characteristic feature of stable secondary structure is its minimal folding energy (MFE) ranging from 37.3 to $59.6(-\mathrm{kcal} / \mathrm{mol})$ for ten in-silico predicted premiRNAs. The MFE of the precursor miRNA was retained low to achieve thermodynamic stability ${ }^{45}$. Owing to their sequence length polymorphism, pre-miRNAs characterization was based only on MFEI (Minimum free energy index) following previous reports ${ }^{22,46}$. The MFEI ranges from 0.57 to 1.03 for ten in-silico predicted pre-miRNAs (Fig. 2). The (A+U) \% of precursor horsegram miRNAs ranges from 39.24-60.38 satisfies the criteria included by Zhang et al. ${ }^{18}$. Nucleotide distribution $(\mathrm{A}=31.6 \%, \mathrm{U}=32.31 \%, \mathrm{G}=26 \%$ and $\mathrm{C}=23.8 \%)$ of the predicted pre- $m i R$ are heterogeneous as given Tables 1 and 3. On an average pre-miRs were of $111.4 \mathrm{bp}$ length and mature miRs ranged from 18 to 24 (Fig. 3a) with mean of 20.73 (Table 3 ). Of predicted $m i R s, m i R 482$ family had more members (six) representing its presence as one of the supreme molecule in the consortia of horsegram regulatory miRs. Similarly, miR 156 and $m i R 390$ were found to have two members each whereas, other miRs have singlets from each family (Fig. 3b). Although, no miRs were identified from ESTs and SSH library validating them as coding sequences, the TSA sequences showed miRNA frequency of 1 in 1937 contigs (Table 4). The results of the predicted miRs and its targets in horsegram were statistically analyzed and summarized in Tables 3 and 4.

Evolutionary relationship with soybean. Soybean (G. max L.), the closely related model legume crop with complete genome information cracked has been used to establish a comparative syntenic map of predicted horsegram miRNAs to determine the putative regions of homologous miR genes. From mapping results, except 


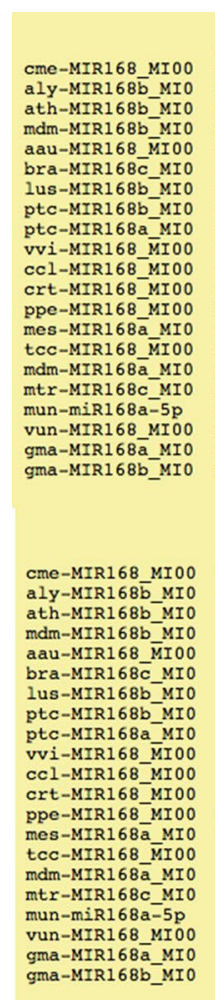

10 20 40 50 60

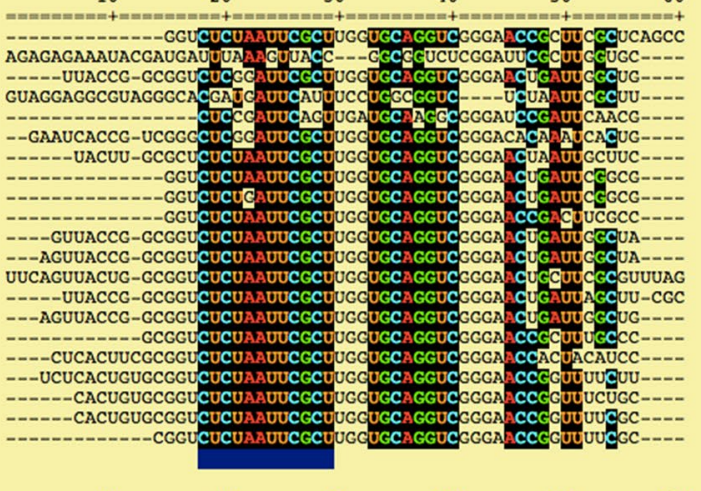
70 80 90 100 110 120

UACGGUUUACGUUUUGUCG-UUGGCCGGUCCUUCUUCAUUUUCCUUUUUUGUUCUUCUCU -AGGUCGGGAACUGAUUAG-CUGACAAUUACACGUGUGUUGUCAUGG------GGUGCAGG-UCGGGAACCGCUUCGYCCCGUUUGAGC----------CUAU - CCGGUAAGGAAACCGUCC-AUUUCGCGCCAUCCGGCGCGGUGGUGG-------

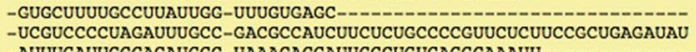
-AUUUGAUUGCCAGAUGGC-UAAACACGAUUGGCUGUGAGGCAAAUU-------DAAAAGGAAG

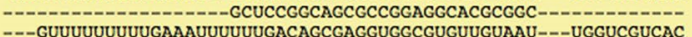
--GUUUUUUUUUGAAAUUUUUUGACAGCGAGGUGGCGUGUUGUAAU---UGGUCGUCAC ---GUUUUUUUUUGAAAUUUUUUGACAGCGAGGUGGCGUGUUGUAAU---UGGUCGUCAC GGCCUUUUUUUCGUACAAGACCUAUGAGCCAGGUGGCGCCAUGGUAUUGGUCGGUG---- CUGCUCCUUAAUAGUGGA-UUGGAUUGGAUUUAGGCCAGAUGGCGG---:------- UACUUUACCGUGUCUGGG-CCUAUGAGCUAGGUGGCUCGAUGU-

---GCUG-UUUUCCUAGAAACCGGCG

---CUGAAAUG-GUGGACCUCUCCCCCGCC-----GCGAAAUG-GUGAAGUGGUAGCCGGCG

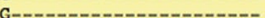
GGCGCCGCCG-

190

200

210

220

cme-MIR168_MI00 aly-MIR168̄ MIO ath-MIR168b MIO mdm-MIR168b-MIO Mau-MTR168 MIOO aau-MIR168_MIO0 bra-MIR168c_MIO
lus-MIR168b-MIO lus-MIR168b_MIO ptc-MIR168b_MIO ptc-MIR168a_MI0 Ccl-MIR168 MI00 Crt-MTR168-MI00 ppe-MIR168-MI00 ppe-MIR168_MI00 mes-MIR168a_MIO mdm-MIR168a mtr-MIR168c MIO mun-miR168a-5p vun-MIR168 MI00 gma-MIR168̄a MIO gma-MIR168b_MI0

CUGGGCGAUUGCAUCCCGCCUUGCAUCAACUGAAUCGGAAACCGUCGG GUCCGAGGUGAAAAAAUAAGGAUCUUACUUUCAGAUCUCCGCGGUGAagGCGGUUUCAUGGAUCUUGCGUUCCUGC

UCGGAGCCUCCAAUGGUGAAAC--.

AUCGG:U

UCGG

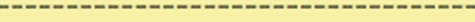

UUCGGAG

AUCGGAG

AUCGGA

UCGGA.

AUCGGAG

UCGGAGACCG

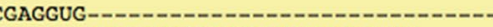

CGAGGUGAGAGAUUGGAUGGAUAUUUACCUU--.---

CCGCGGUGAAGAG---_---

CCGCGGUGA-

UCGGACEC

CGCGGUGAAUCUA-_-_-_-

(-)

UCGG:

UCGGAGGCCGCGGUGAAC-

GGCCG-

130

140

150

160

70

180

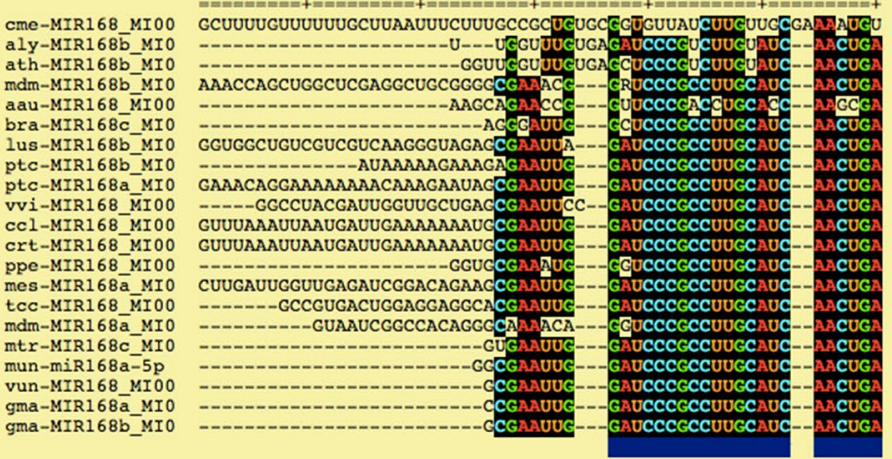

Figure 5. Pre-miR sequence conservation blocks for miR168 family of horsegram with related plants. Non-coding RNAs are conserved across species with specific role in development, metabolism and energy conservation. 


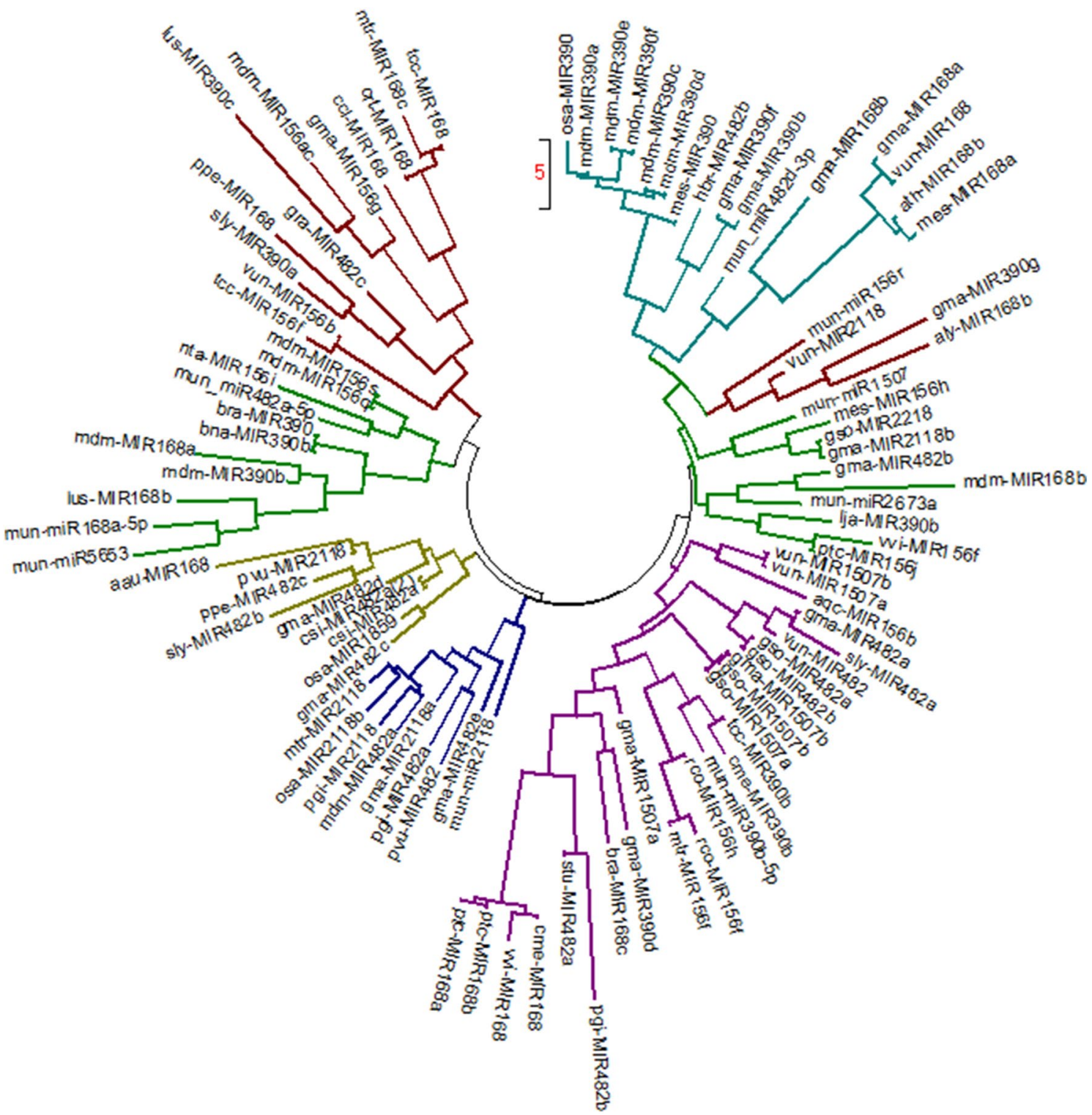

Figure 6. Phylogenetic analysis were done using MEGA $7^{30}$ for predicted miRNA precursors of horsegram with their closely related plant $m i R s$. Nine clusters of miR precursors clustered with related plant species.

mun- $m i R-482 \mathrm{a}-3 \mathrm{p}$ and $m i R 2673 \mathrm{a}$, rest of the putative horsegram miRNAs have orthologs widespread in G. max genome. $m i R 156 \mathrm{r}$ and $m i R 156 \mathrm{k}$ had more number of orthologs. The mapped miRs are depicted in soybean genome (Fig. 4). Comparative genomics enabled us to infer $m i R$ gene function, which could enumerate future research focus contributed by horsegram miRNAs in related species and in distant crops as well.

Conservation and phylogenetic analysis. The pre-miR homologs were identified by performing blast of horsegram pre-miRNA against the $m i R$ Base database. The hits were filtered to retrieve only miRNAs of same family. In this study, high degree of conservation was exemplified by comparison of mun-miR168 to other plant precursor miRNAs (Figs. 5 and 6). It is evident that horsegram miRNAs share similarity with related legume species (Fig. 7). It is conclusively apparent that, horsegram miRNAs seem to have evolved at different rates in different time period similar to other plants.

Putative target genes, their functions and networks. Inferring the function of $m i R N A$ targets is crucial to significantly substantiate the functional role of $m i R N A$ s in gene expression and regulation. For predicted 15 horsegram miRs, 39 target genes were identified and classified into different groups based on their functional annotation. As revealed by Mercator annotation results (Fig. 8) of predicted $m i R$ targets, diverse processes ranging from RNA transcription regulation, protein posttranscriptional modifications, development, signalling, biotic and abiotic stress tolerance and glycolysis were being regulated by the identified $15 \mathrm{miRs}$. In addition, target genes also regulate cell wall degradation, hormone biosynthesis and redox components like ascorbate 


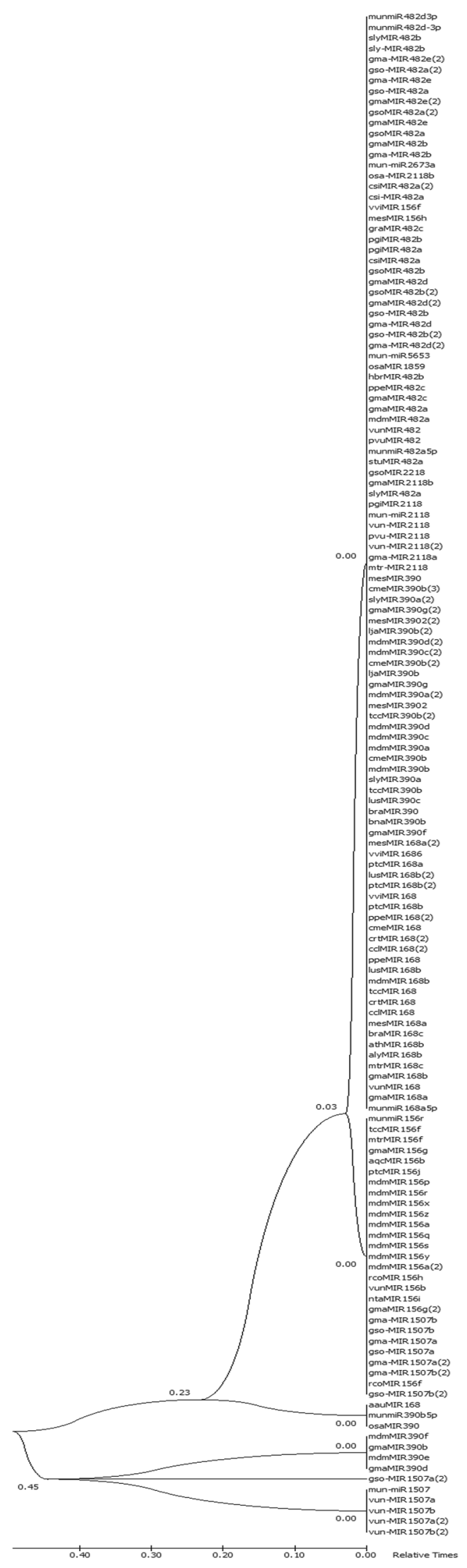

Figure 7. Phylogenetic tree of mun- $m i R$ families were constructed using PhyML $^{40}$ and TreeDyn ${ }^{41} \cdot m i R$ family 1507 is having related species $m i R$ in a separate cluster and their role in stress tolerance is also unique. 


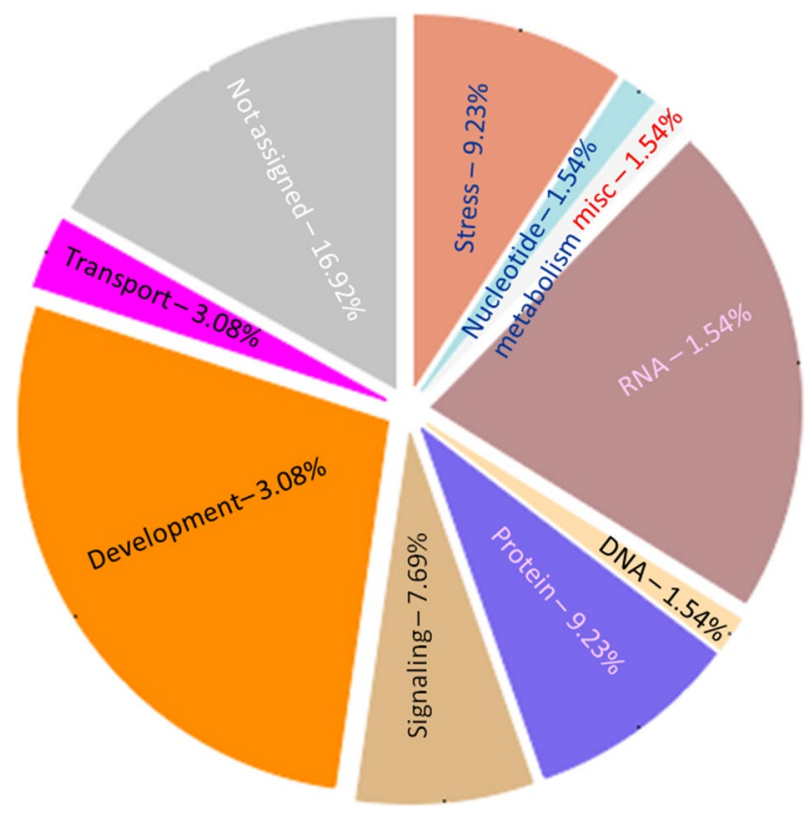

Figure 8. Mercator annotation results. Functional groups of identified transcripts based on annotation results drawn using Mercator ${ }^{42}$. Predicted $m i R$ targets ranging from RNA transcription regulation, protein post transcriptional modifications, development, signalling, biotic and abiotic stress tolerance and glycolysis were being regulated by the identified 15 miRs.

and glutathione synthesis (Table 5). The annotation of target genes using the Mercator tool categorized them into12 broad biochemical processes (Fig. 9). Differential expression of drought responsive miRNAs and gene networks Transcriptome analysis of Illumina sequence data from eight samples representing shoot and root tissues of contrasting horsegram genotypes M-191 (drought sensitive) and M- 249 (drought tolerant) (NCBI Bioproject PRJNA216977) was used to decipher the horsegram miRNA expression in root and shoot samples each under normal and drought stress condition respectively ${ }^{11}$. The miRNA abundance estimated from blast output indicates the behavioral miRNA expression (Fig. 10). The clustering simplifies the differential gene expression levels of horsegram miRNA and samples based on the similar expression. Network predicted and depicted as target- target interaction (Fig. 11) designates machineries of energy conservation confirming earlier hypothesis of structural compaction and energy conservation to survive under stress conditions ${ }^{14,15}$. This hypothesis may best fit and appropriate for other crops as well. To extend this hypothesis we identified target homologs in other plants also (Fig. 12).

\section{Discussion}

Comprehensive studies on plant miRNAs endorse stage specificity and multitude of targets ${ }^{47-49}$. The non-coding $m i R N A s$ play a regulatory role in its target protein coding gene expression ${ }^{50}$. The miRNA multiplexes with $R N A$ induced silencing complex (RISC) guiding the repression or cleavage of its target messenger RNA by seed nuclei base-pairing ${ }^{3}$. Complementarity between $m i R N A$ s and their target genes are high to regulate developmental processes, metabolism and stress responses ${ }^{51,52}$. Hence, there is enormous necessity to identify and validate miRNAs for further downstream applications in plants $^{22}$. In horsegram like deficit moisture stress tolerant crop, major genes interaction network influence in expression of a tolerant phenotype. From contrasting stress responsive genotype data, and qPCR of identified miRNAs from a single stress responsive genotype under irrigated and deficit stress (Fig. 13), we could identify differential expression of $m i R$ genes. These predicted 15 miRs were clustered in to 11 different families and are conserved. To validated identified miRNAs qPCR was performed as reported earlier (53). Of the total eight miRs validated, two clusters were formed. Mun-miR 482 was found in both the clusters; whereas, mun-miR1507 was found in one cluster which distinguish the tested variety Paiyur1 for its ability to react for stress (Fig. 14). Of the 15 identified mun-miRs, miR 156, miR 171 and miR 390 were found to be differentially expressed in germinating seeds of halophyte Reaumuria soongorica under salt stress conditions (54). Legume genomes are rich in SNPs. Saturated map of SNPs were reported in legumes like vigna (55) and In pigeonpea (56). Of which highest haplotype desnsity of 0.7380 was reported for serine threonine kinase coding disease resistance gene (56) which was identified as an important miR target in the present ivestigation. Variations in these miR sequences are expected among germplasm to change their expression. This could be a reason for non expression of other genes. The conserved nature of maximum plant miRNAs bolstered the miRNA search in different plant species utilizing available genomic resources like ESTs, GSS and $m R N A$ sequences ${ }^{34,57-59}$ 


\begin{tabular}{|c|c|c|}
\hline miRNA_Acc & Target_Acc & Target_Description \\
\hline \multirow{7}{*}{ mun-miR482d-3p } & AT2G28380.1 & DRB2/dsRNA-binding protein 2 \\
\hline & AT2G36470.1 & Plant protein of unknown function (DUF868) \\
\hline & AT4G06560.1 & Transposable element gene \\
\hline & AT3G30713.1 & Transposable element gene \\
\hline & AT5G65700.1 & BAM1/Leucine-rich receptor-like protein kinase family protein \\
\hline & AT5G65700.2 & BAM1/Leucine-rich receptor-like protein kinase family protein \\
\hline & AT4G06587.1 & Transposable element gene \\
\hline \multirow{12}{*}{ mun-miR482b-5p } & AT1G78270.1 & AtUGT85A4, UGT85A4/UDP-glucosyl transferase 85A4 \\
\hline & AT2G32700.5 & LUH/LEUNIG_homolog \\
\hline & AT2G32700.2 & LUH/LEUNIG_homolog \\
\hline & AT2G32700.4 & LUH/LEUNIG_homolog \\
\hline & AT2G32700.6 & LUH/LEUNIG_homolog \\
\hline & AT2G32700.1 & LUH/LEUNIG_homolog \\
\hline & AT2G32700.7 & LUH/LEUNIG_homolog \\
\hline & AT1G48550.1 & Vacuolar protein sorting-associated protein 26 \\
\hline & AT1G48550.2 & Vacuolar protein sorting-associated protein 26 \\
\hline & AT5G28650.1 & WRKY74, ATWRKY74/WRKY DNA-binding protein 74 \\
\hline & AT3G58640.2 & Mitogen activated protein kinase kinase kinase-related \\
\hline & AT3G58640.1 & Mitogen activated protein kinase kinase kinase-related \\
\hline \multirow{4}{*}{ mun-miR482a-3p } & AT1G72050.2 & TFIIIA/transcription factor IIIA \\
\hline & AT1G72050.1 & TFIIIA/transcription factor IIIA \\
\hline & AT4G03080.1 & BSL1/BRI1 suppressor 1 (BSU1)-like 1 \\
\hline & AT5G12000.1 & Protein kinase protein with adenine nucleotide alpha hydrolases-like domain \\
\hline mun-miR482a-3p & AT1G22930.2 & T-complex protein 11 \\
\hline \multirow{13}{*}{ mun-miR156r } & AT2G16000.1 & Transposable element gene \\
\hline & AT3G45775.1 & Transposable element gene \\
\hline & AT2G43370.1 & RNA-binding (RRM/RBD/RNP motifs) family protein \\
\hline & AT5G50570.1 & $\begin{array}{l}\text { SPL13A, SPL13/Squamosa promoter-binding protein-like (SBP domain) transcription factor family } \\
\text { protein }\end{array}$ \\
\hline & AT5G50570.2 & $\begin{array}{l}\text { SPL13A, SPL13/Squamosa promoter-binding protein-like (SBP domain) transcription factor family } \\
\text { protein }\end{array}$ \\
\hline & AT2G42200.1 & SPL9, AtSPL9/squamosa promoter binding protein-like 9 \\
\hline & AT5G50670.1 & $\begin{array}{l}\text { SPL13B, SPL13/Squamosa promoter-binding protein-like (SBP domain) transcription factor family } \\
\text { protein }\end{array}$ \\
\hline & AT3G57920.1 & SPL15/squamosa promoter binding protein-like 15 \\
\hline & AT1G27370.1 & SPL10/squamosa promoter binding protein-like 10 \\
\hline & AT1G69170.1 & Squamosa promoter-binding protein-like (SBP domain) transcription factor family protein \\
\hline & AT1G27370.2 & SPL10/squamosa promoter binding protein-like 10 \\
\hline & AT5G43270.1 & SPL2/squamosa promoter binding protein-like 2 \\
\hline & AT1G27370.4 & SPL10/squamosa promoter binding protein-like 10 \\
\hline \multirow{10}{*}{ mun-miR1507a } & AT2G15970.2 & COR413-PM1 | cold regulated 413 plasma membrane 1 \\
\hline & AT4G10465.1 & Heavy metal transport/detoxification superfamily protein \\
\hline & AT2G15970.1 & $\begin{array}{l}\text { COR413-PM1, WCOR413, WCOR413-LIKE, ATCOR413-PM1, FL3-5A3, ATCYP19 |/cold regulated } \\
413 \text { plasma membrane } 1\end{array}$ \\
\hline & AT4G06678.1 & Transposable element gene \\
\hline & AT5G35142.1 & Transposable element gene \\
\hline & AT5G44925.1 & Transposable element gene \\
\hline & AT3G21250.1 & ATMRP6, MRP6, ABCC8 | multidrug resistance-associated protein 6 | \\
\hline & AT3G21250.2 & MRP6, ABCC8 | multidrug resistance-associated protein 6 | \\
\hline & AT3G54510.2 & Early-responsive to dehydration stress protein (ERD4) \\
\hline & AT3G54510.1 & Early-responsive to dehydration stress protein (ERD4) \\
\hline \multirow{3}{*}{ mun-miR168a-5p } & AT1G48410.3 & AGO1/Stabilizer of iron transporter SufD/Polynucleotidyl transferase \\
\hline & AT1G48410.1 & AGO1/Stabilizer of iron transporter SufD/Polynucleotidyl transferase \\
\hline & AT1G48410.2 & AGO1/Stabilizer of iron transporter SufD/Polynucleotidyl transferase \\
\hline
\end{tabular}




\begin{tabular}{|c|c|c|}
\hline miRNA_Acc & Target_Acc & Target_Description \\
\hline \multirow{8}{*}{ mun-miR2118 } & AT2G21230.2 & Basic-leucine zipper (bZIP) transcription factor family protein \\
\hline & AT2G21230.1 & Basic-leucine zipper (bZIP) transcription factor family protein \\
\hline & AT2G21230.3 & Basic-leucine zipper (bZIP) transcription factor family protein \\
\hline & AT5G45050.2 & TTR1, ATWRKY16, WRKY16/Disease resistance protein (TIR-NBS-LRR class) \\
\hline & AT5G45050.1 & TTR1, ATWRKY16, WRKY16/Disease resistance protein (TIR-NBS-LRR class) \\
\hline & AT3G05680.1 & EMB2016/embryo defective 2016 \\
\hline & AT3G05680.2 & EMB2016/embryo defective 2016 | \\
\hline & AT1G50120.1 & Unknown function/Expressed \\
\hline \multirow{8}{*}{ mun-miR482a } & AT5G15850.1 & COL1, ATCOL1/CONSTANS-like 1 \\
\hline & AT1G74600.1 & Pentatricopeptide (PPR) repeat-containing protein \\
\hline & AT5G15840.1 & CO, FG/B-box type zinc finger protein with CCT domain \\
\hline & AT5G15840.2 & CO, FG/B-box type zinc finger protein with CCT domain \\
\hline & AT2G21230.2 & Basic-leucine zipper (bZIP) transcription factor family protein \\
\hline & AT2G21230.1 & Basic-leucine zipper (bZIP) transcription factor family protein \\
\hline & AT2G21230.3 & Basic-leucine zipper (bZIP) transcription factor family protein \\
\hline & AT1G18300.1 & atnudt4, NUDT4/nudix hydrolase homolog 4 \\
\hline \multirow{3}{*}{ mun-miR390b-5p } & AT1G58050.1 & RNA helicase family protein \\
\hline & AT5G67610.1 & Uncharacterized conserved protein (DUF2215) \\
\hline & AT5G67610.2 & Uncharacterized conserved protein (DUF2215) \\
\hline
\end{tabular}

Table 5. Functional annotation of horsegram miRNA targets.

to a great extent and we followed the trend in identifying them in a drought tolerant crop. Conserved miRNAs in ginger, garlic, coffee and tea were identified ${ }^{60-63}$. For predicted pre-miRNAs, MFEI resolution for length variation extends from 0.57 to 1.03 in horsegram. Pre-miRs illustrate the absence of large internal loops/bulges and at least 20 nucleotides for Wobble base pairing (G/U base pairings) or Watson-Crick base pairing between the miRNA and the star sequence ${ }^{62,64}$. Estimated $(\mathrm{A}+\mathrm{U}) \%$ of pre-miRs range satisfies the predetermined criteria ${ }^{18}$. The putative genomic region of these non-coding sequences were determined and intergenic sequences were utilized for secondary structure prediction with the stringent filtering criteria to attain potential precursor $m i R N A^{26}$. Resultant secondary structures were inspected for precursor miRNA and the positioning of the mature miRNA within its stem-loop structure manually. The sequences with suitable secondary structure ${ }^{66}$ were characterized for structure attributes and its possible existence as potential precursor of the predicted miRs. The transcription of miRNAs from sense and antisense strands of genes were already reported ${ }^{67}$. Our results stand by the possibility of the miRNA in both sense and antisense strands (Table 2) similar results were already reported in potato, tobacco and B. rapa ${ }^{16,68,69}$. The frequency of miRNA using expressed sequence tags is 1 in 1000 confirming previous reports ${ }^{62}$. The interaction of $m i R N A s$ with its target genes can enumerate evolutionary role of microRNAs ${ }^{48,70,71}$. Utilization of miRNAs in RNA interference (RNAi) mediated gene regulation has emerged as an important tool in novel traits engineering either by over expression of a miRNA or target genes by synthetic miRNAs. Gene silencing/knockout may help in understanding miRNA in plant responses to stress resulting increased productivity with improved nutritional value ${ }^{72}$. Cellular functions were not known for eight of the target proteins identified in the present investigation. Biotic/Abiotic stresses are critically affecting growth and development of plants. There are reports on contrasting mechanisms confirming grades of deficit moisture stress condition to control among different horsegram germplasm sources ${ }^{6,15}$. Among the identified miRNAs mun$m i R 156 \mathrm{r}$, mun-miR156k, mun-miR482a, mun-miR390b-5p and mun-miR482a-5p were noticed to be involved in RNA processing, protein synthesis and modifications as well as plant development by altering the expression of their respective targets, whereas the genes encoding abiotic stress, biotic stress (NBS-LLR resistant class) and signalling associated proteins were targeted by mun-miR482a-5p, mun-miR482d-3p and mun-miR1507a respectively. Thus, there is concurrent substantiation that microRNAs can play pivotal role in crop improvement ${ }^{73}$ and the miRNAs predicted from this investigation can be useful for future research. Closely related homologs of predicted putative $m i R s$ exhibited high degree of conservation as in mum-miR482 with related plant mature $m i R N A s$ (Fig. 6). The precursor sequences from $m i R 482$, $m i R 390$ and $m i R 150$ represented a strong candidate promulgating its necessary importance at post-transcriptional gene regulation in horsegram. The conserved nature of precursor and mature miRNAs has been reported in various plant groups from earlier studies ${ }^{16,34,73,74}$. The potential of $m i R N A$ s to bind corresponding target $m R N A$ are comparable with their complementarity to degrade target $m R N A$. Therefore, to infer the contribution of microRNAs in cellular functions and regulatory gene networks, the miRNA target gene prediction is a crucial step ${ }^{64}$. The majority of the predicted miRNA targets in horsegram depict energy conservation mechanism which play important role in survival during adverse 


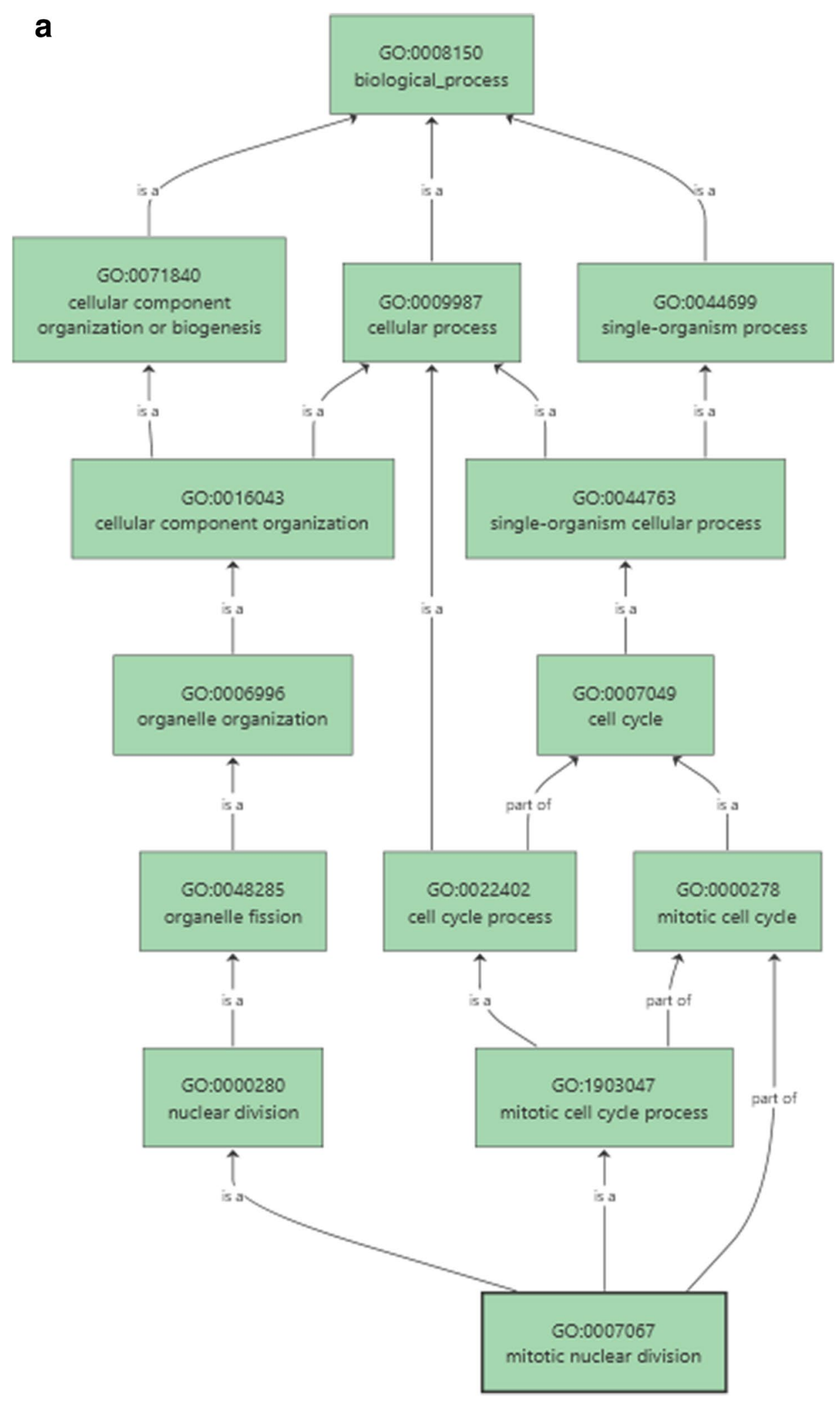

Figure 9. Target groups based on functional groups identification from $\mathrm{B} 2 \mathrm{~g}^{44}$. (a) Cell cycle, cell division and basic cell process were the key functions predicted among the $m i R$ target sequences. (b) Cell intergrity and intact cell membrane were being targeted indicating the activation of degradation pathways under stress conditions. 


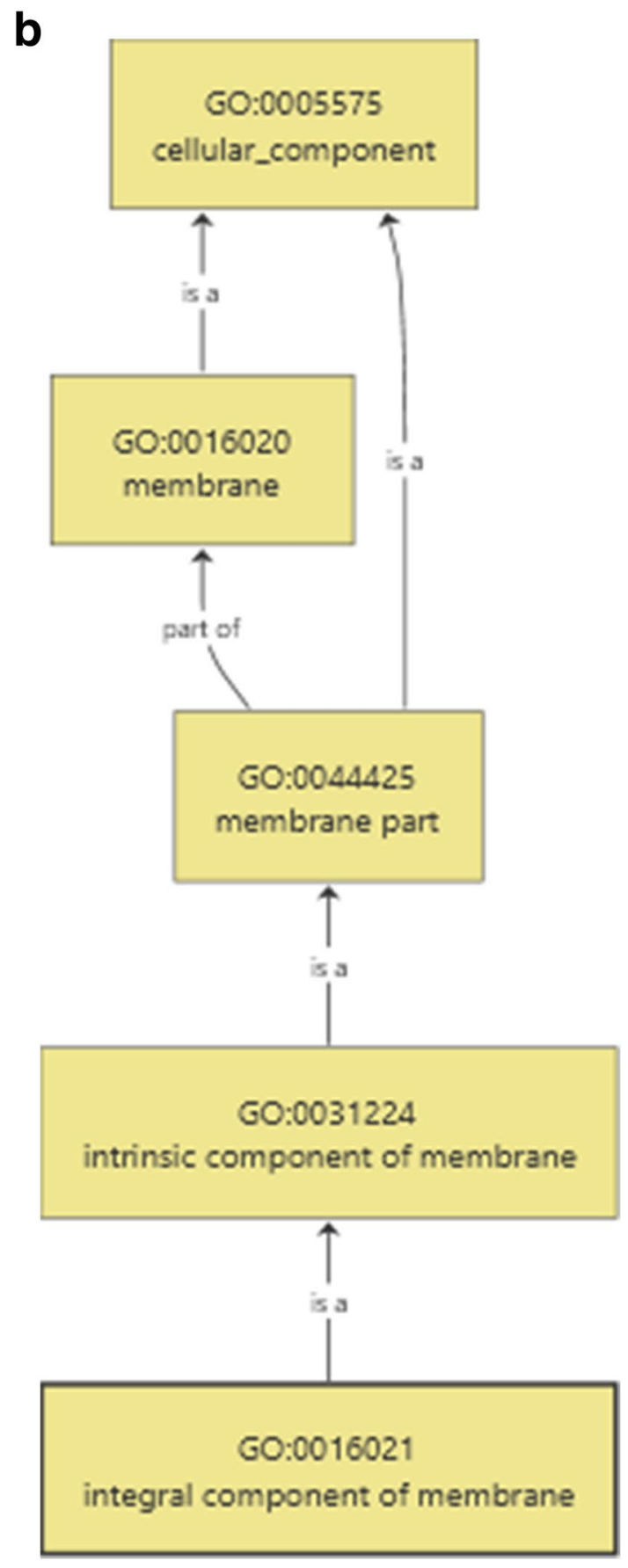

Figure 9. (continued) 

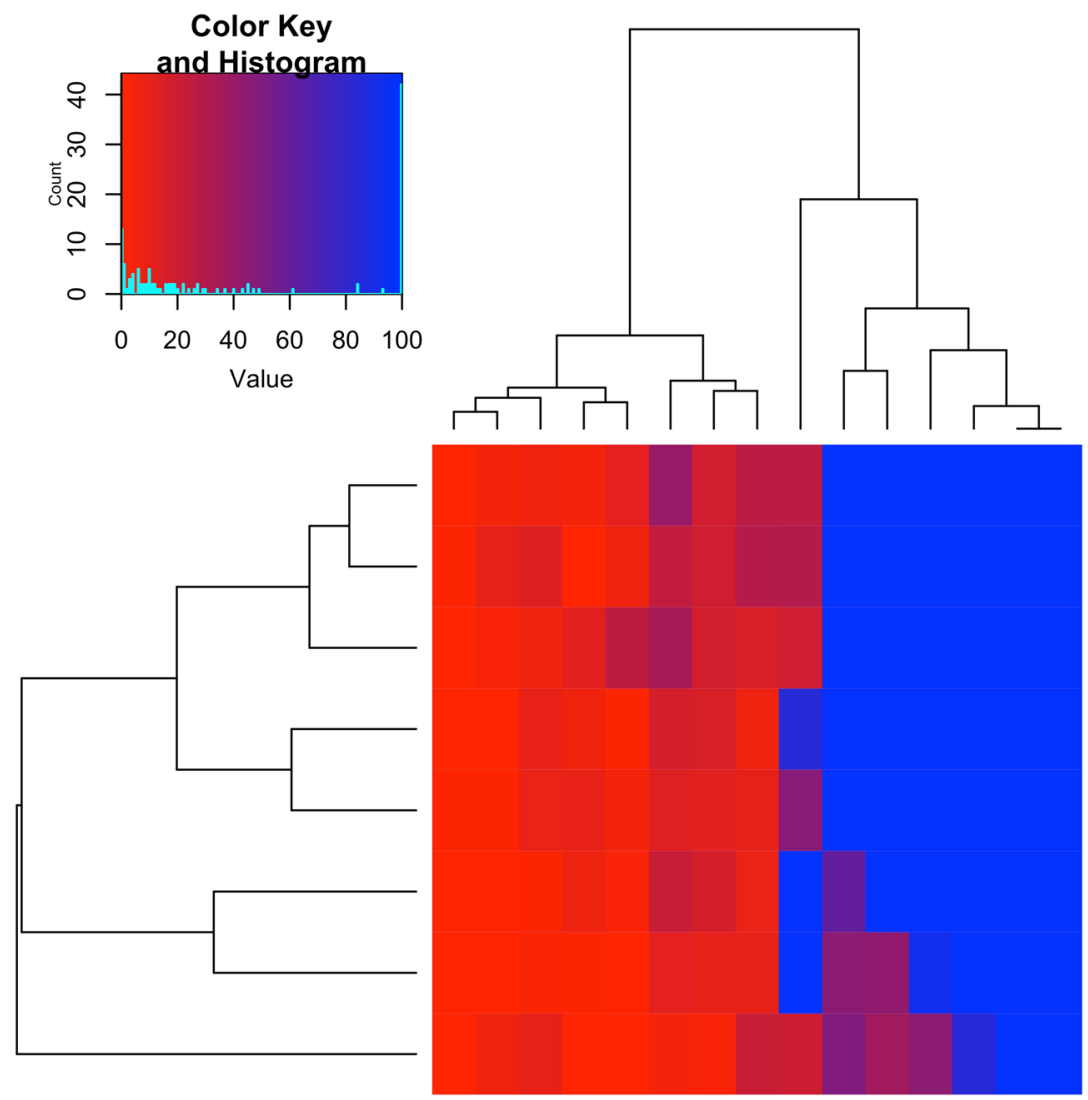

RtM249(D)

RtM191(C)

RtM191(D)

StM249(C)

StM191(C)

StM191(D)

StM249(D)

$\operatorname{RtM} 249(C)$

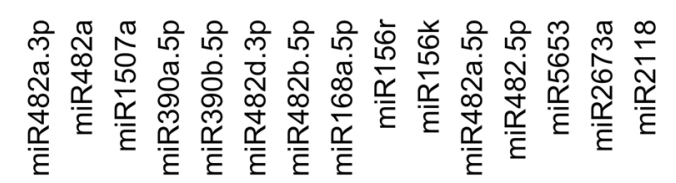

Figure 10. Expression heatmap of putative miRNAs differential expression in horsegram. Expression analysis clearly indicated two clusters of miRs. There is enough variation among the source tissue and gene families. The results of in-silico and real time quantitative expression pattern of $m i R$ are similar confirming prediction efficiency of the pipeline.

conditions. Target gene prediction reveals the regulation of multiple genes by single miRNA with different levels of regulation ${ }^{22,47,75}$. Also, the genes targeted belong to more than one gene family, which shows the multitude of miRNA functions in various metabolic processes (Table 4). We could spot 39 target genes of predicted miRs in horsegram and its homologs in other plant species. This indicates that, drought tolerance phenotype can be manipulated by adjusting the expression of identified miRs and their targets.

\section{Conclusions}

Great concern is being publicized recently to unravel various mechanisms involved in drought tolerance with emphasis to changing climatic conditions. Even though, there are extensive inquiries on $m i R N A$ s discovery and their functional prediction were completed, some of the non-model plants with considerable traits were not subjected to systematic investigation to elucidate contrasting mechanisms and their key players. Eight novel miRNAs were $^{76}$ predicted from horsegram. However, these non-validated miRNAs were predicted with low stringency and standard nomenclature was not followed. Inappropriately, the drought hardy horsegram miRNAs still remain unknown and lacks extensive validation. In this study, 15 conserved miRNAs belonging to nine different families 


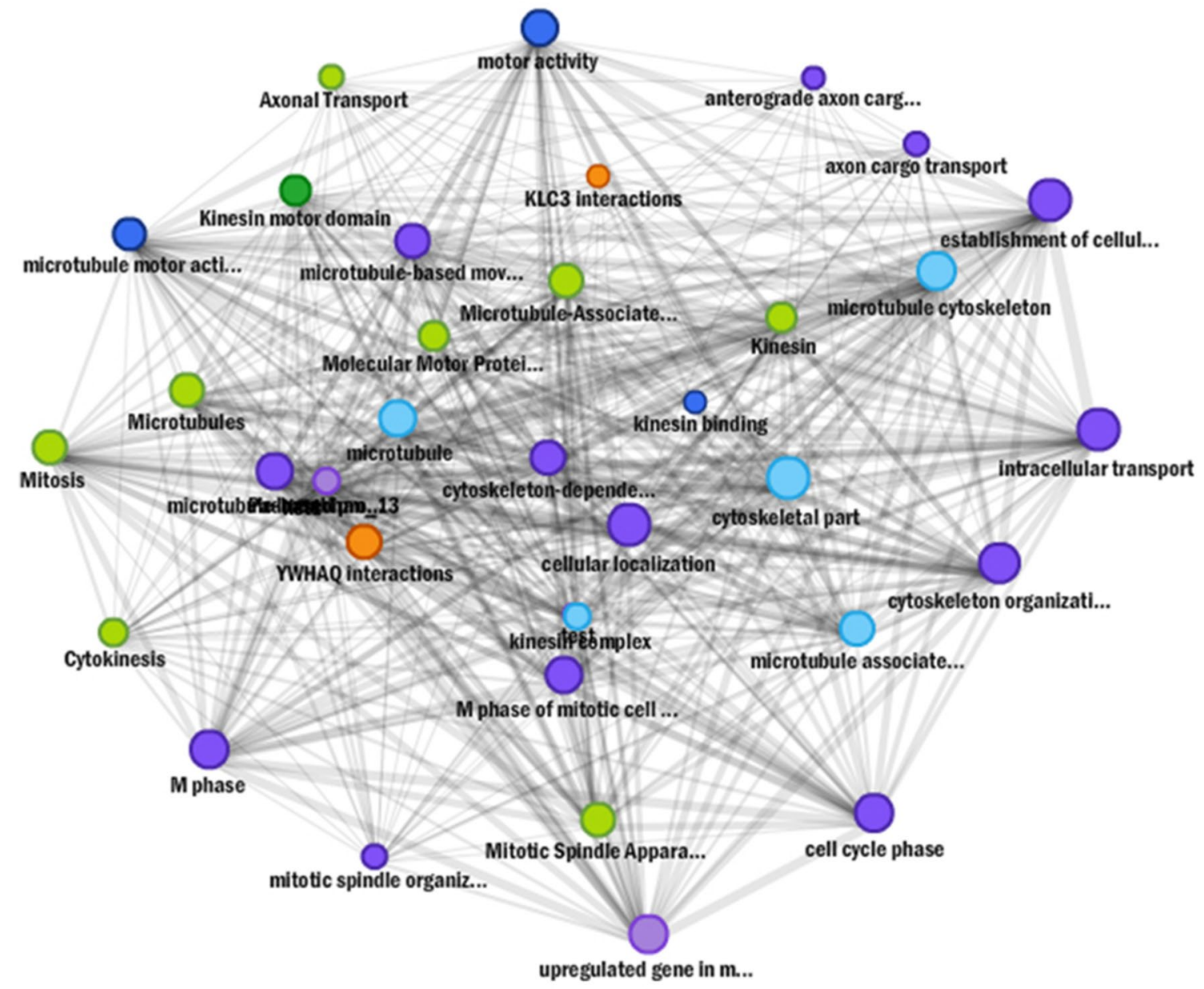

Figure 11. Predicted miRNA target genes function network. The function based protein target network drawn using sting server ${ }^{82}$ elucidates the role of $m i R s$ identified in cell development and maintenance of minimal functions during stress conditions. Most of the reductions in functions identified were correlated to energy conservation mechanism.

were identified from EST and TSA sequences and validated in this first report with its tissue specific differential expression (Fig. 10). Mun-miR482a-5p and mun-miR482d-3p are $m i R 482$ family $m i R N A$ s reported to regulate stress response in soybean and apple $\mathrm{e}^{77,78}$. The same trend is observed in our results confirming the earlier report. Mun-miR390b-5p is a $m i R 390 \mathrm{~b}$ family $m i R N A$ earlier found for protein degradation and post transcriptional modifications in Arabidopsis, maize and soybean ${ }^{79-81}$. Upregulation of miR390b and downregulation of 1507 family miRNA was observed (Fig. 14) in qPCR of Paiyur1 variety which is a stress responsive accession. The present investigation indirectly links stress tolerance to energy conservation as indicated by the network rather than direct response to stress conditions (Fig. 15). Hence, we confirm the interplay of miRNA-stress responseNBS-LLR class R-protein response ${ }^{77}$ in energy conservation to survive the stress conditions. Additionally, the study reveals that identified miRNA regulated target genes have differential biological functions including cell wall degradation, hormone synthesis and synthesis of redox component like ascorbate and glutathione (Table 5). These findings can further help in translational research of related legumes and may result in selection of better germplasm for higher productivity and nutritional security. The network predicted from this investigation confirms the earlier hypotheses: structural compaction to overcome stress tolerance ${ }^{14,15}$. Altogether, the outcomes of the present investigation deliver clarity that, horsegram is a drought adapted crop and can be considered as a model crop for drought tolerance research. Thus, the predicted horsegram miRNAs may unravel the unique 


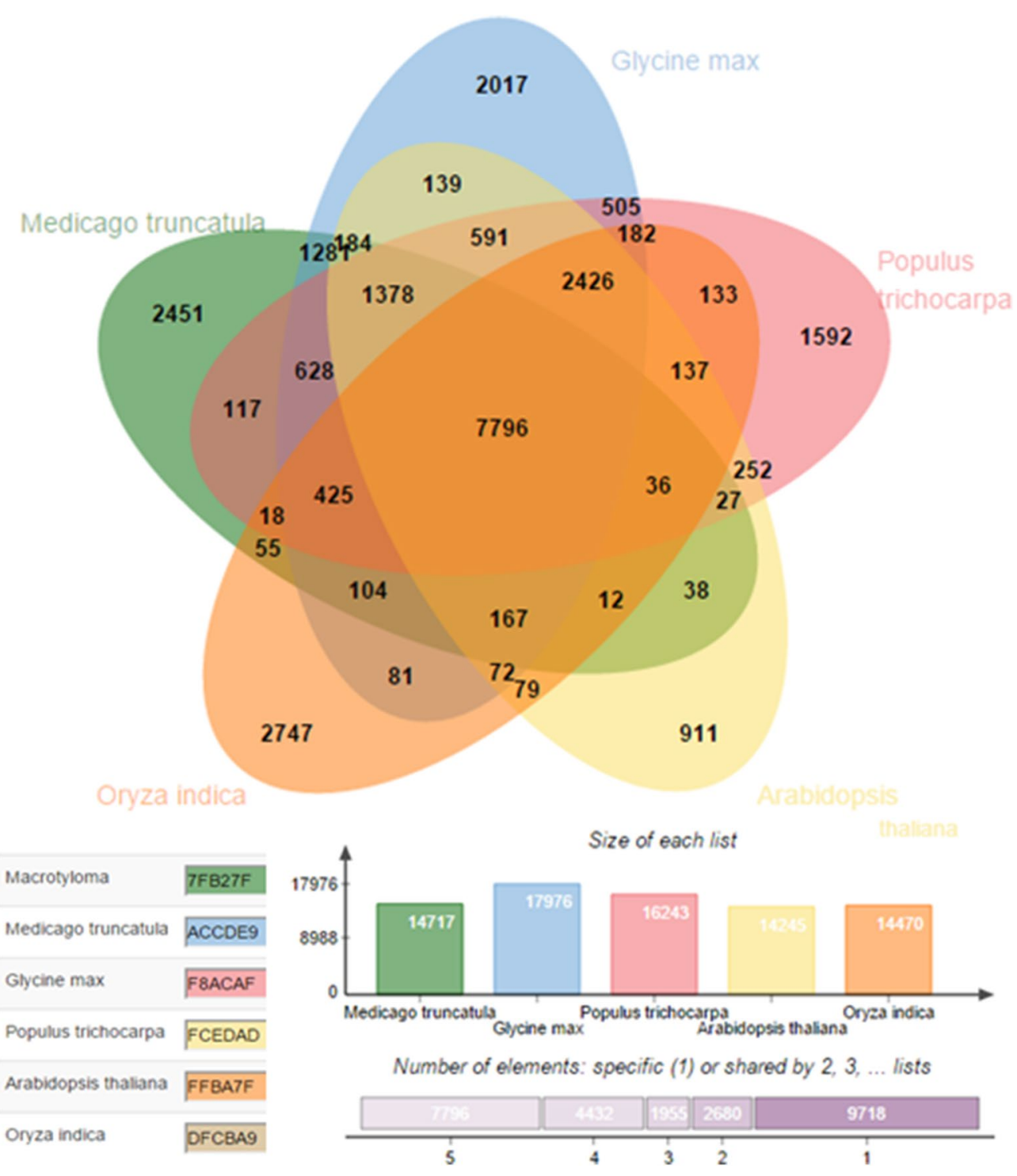

Figure 12. Predicted $m i R$ target whole genome wide homologs across species were identified using OrthoVenn $2^{83}$. Like the conserved $m i R s$ across species, the target range is also conserved in different plant species indicating existence of similar mechanism.

tolerance capability associated to its metabolic pathways and the present workflow represents simple and straightforward approach for the prediction and characterization of miRNAs in those plants for which genomes are yet to be splintered. 


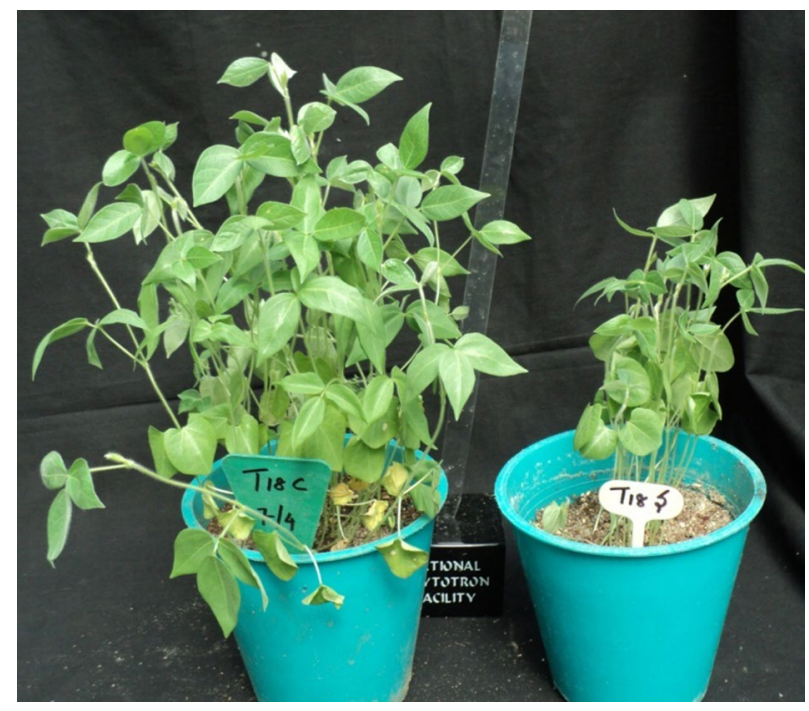

Figure 13. Control and stress treatments of horsegram plants. Paiyur1 plants were grown under glass house conditions. C. control plant pot was maintained with frequent recharging of soil moisture by irrigation; $\mathrm{S}$. Plants grown under severe soil moisture deficit condition. Day time the plants suffered temporary wilting and regains during evening hours.
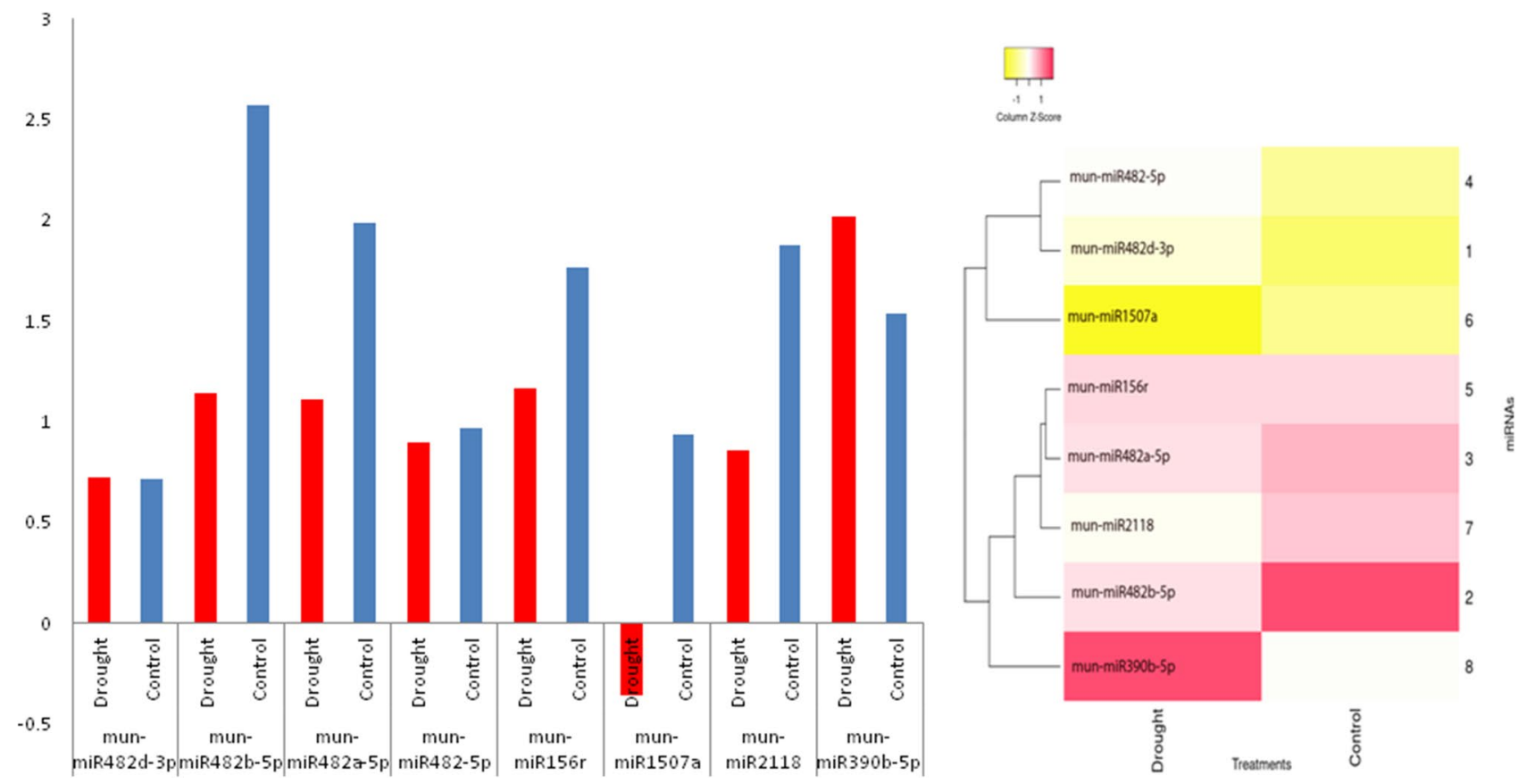

Figure 14. Quantitative rtPCR based differential expression of identified mun-miRs. Heatmaps were generated using Heatmapper ${ }^{84}$. Quantitative expression of miRs formed two clusters. Expression of miR1507 and miR390 families defines Paiyur1 phenotypic response to stress conditions. Down-regulation of other family miRs could be correlated to decline in cellular functions under stress conditions. 

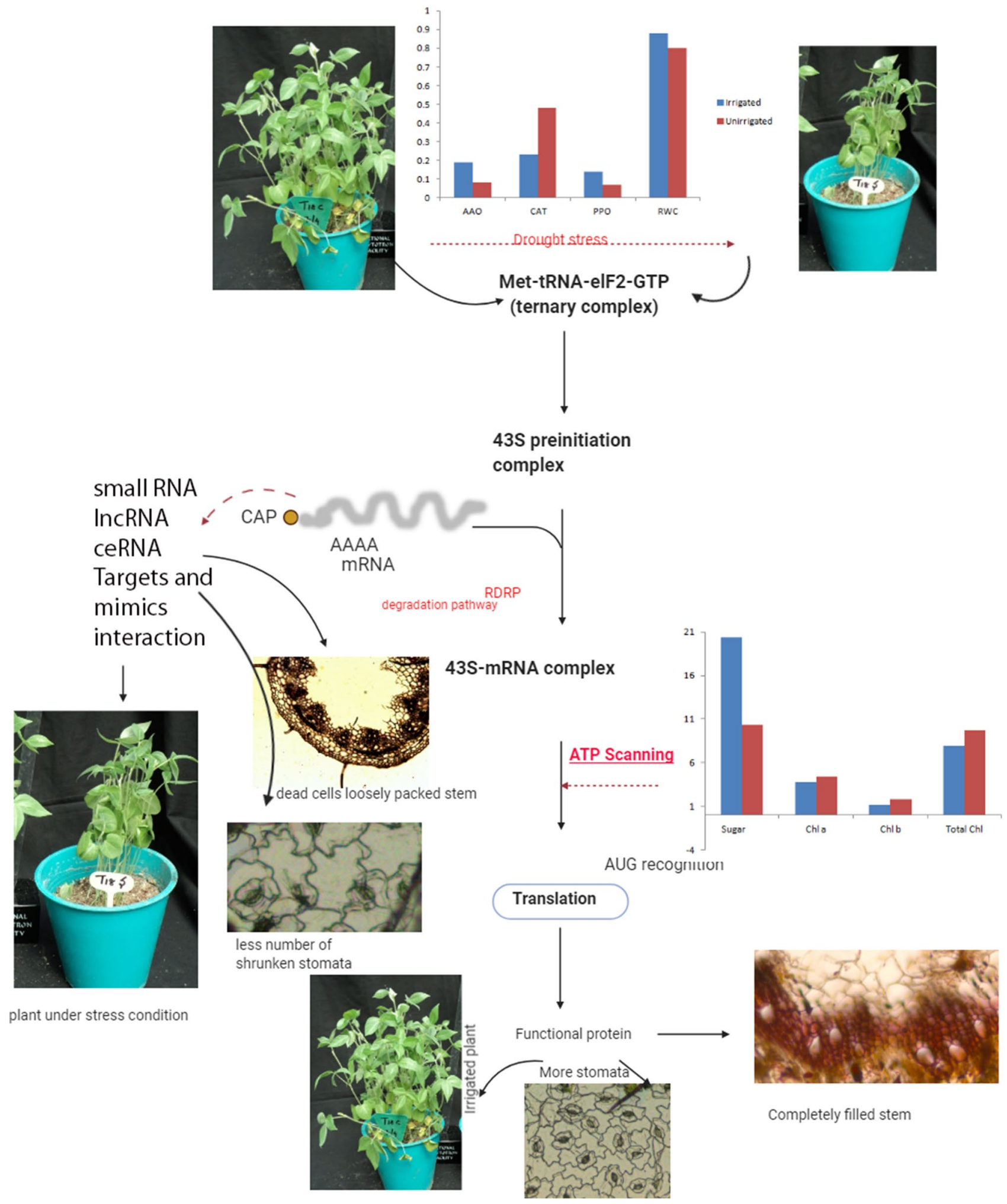

Figure 15. Control and stress treatments of horsegram plants and perspective conclusion of the predicted stress tolerance mechanism. Illustration was drawn with wet lab results and glass house grown plant sample analyses. Under stress conditions AAO expression defines the tolerance with declined total sugar and chlorophyll contents. Pith autolysis and shrunken stomata was observed under soil moisture deficit stress. 
Received: 16 March 2020; Accepted: 14 September 2020

Published online: 14 October 2020

\section{References}

1. Jones-Rhoades, M. W., Bartel, D. P. \& Bartel, B. MicroRNAs and their regulatory roles in plants. Annu. Rev. Plant Biol. 57, 19-53 (2006).

2. Pritchard, C. C., Cheng, H. H. \& Tewari, M. MicroRNA profiling: approaches and considerations. Nat. Rev. Genet. 13, 358 (2012).

3. Bartel, D. P. MicroRNAs: genomics, biogenesis, mechanism, and function. Cell 116, 281-297 (2004).

4. Arora, R. K. \& Chandel, K. P. S. Botanical source areas of wild herbage legumes in India. Trop. Grasslands. 6, 213-221 (1972).

5. Yasin, J. K. et al. Identification and validation of genes responsible for moisture stress tolerance in Horsegram (Macrotyloma uniflorum(Lam.) Verdc.), LIBEST_027585.2011. https://www.ncbi.nlm.nih.gov/biosample/SAMN00750282 (2011).

6. Yasin, J. K. et al. Alternate antioxidant defence system in moisture stress responsive accessions of horse gram. Legume Res. 37, 145-154 (2014).

7. Cook, B.G., Pengelly, B.C., Brown, S.D., Donnelly, J.L., Eagles, D.A., Franco, M.A., Hanson, J., Mullen, B.F., Partridge, I.J. \& Peters, M. Tropical Forages: An interactive selection tool (2005).

8. Morris, J. B. Macrotyloma axillare and M. uniflorum: descriptor analysis, anthocyanin indexes, and potential uses. Genet. Resour. Crop Evol. 55, 5-8 (2008).

9. Peshin, A. \& Singla, S. K. Anticalcifying properties of Dolichos biflorus(horse gram) Seeds. Indian J. Exp. Biol. 32, 889-891 (1994).

10. Reddy, P. C. O. et al. Identification of stress-induced genes from the drought tolerant semi-arid legume crop horsegram (Macrotyloma uniflorum (Lam.) Verdc.) through analysis of subtracted expressed sequence tags. Plant Sci. 175, 372-384 (2008).

11. Bhardwaj, J. et al. Comprehensive transcriptomic study on horse gram (Macrotyloma uniflorum): De novo assembly, functional characterization and comparative analysis in relation to drought stress. BMC Genomics 14, 1 (2013).

12. Yasin, J.K., Mishra, B. K., Chaudhary, S., Magadum, S., Chinnusamy, V. \& Singh, N.K. Transcriptome analyses for genome wide identification of ncRNAs and miRNA from Pigeonpea (Cajanus cajanL.). In "Plant Biology 2016" 13-18th July, 2016 by ASPB, Austin, Texas, USA (2016).

13. Yasin, J.K., Sreevathsa, R., Vivek, T., Nager, R., Lal, S.K., Pillai, M.A. \& Chinnusamy, V. IncRNA plays a major role in Susceptibility to resistance: Insights into pigeonpea RGA. In "Plant Biology 2016" 13-18th July, 2016 by ASPB, Austin, Texas, USA (2016).

14. Yasin, J.K. \& Magadum, S. Structural compaction to conserve energy: ncRNA expression directs $\mathrm{pH}$ flux of floral parts and yield loss in pigeonpea (Cajanus cajanL.). In Royal Society Theo murphy meeting on "Evolution brings Ca2+ and ATP together to control life and death. March 16-17th 2016 at Royal Society of UK, London (2016).

15. Yasin, J.K., Bhat, K.V., Rajkumar, S., Subalakshmi, Ramya, K.T. \& Fiyaz, A.R. Structural compaction, Mechanism of acid tolerance in moisture stress responsive accessions of horse gram. In The 8TH International symposium on "Plant soil interactions at low pH", October 18-22, 2012, Bangalore, India (2012); 170

16. Dhandapani, V. et al. Identification of potential microRNAs and their targets in Brassica rapa L. Mol. Cells. 32, 21-37 (2011).

17. Xie, F. L. et al. Computational identification of novel microRNAs and targets in Brassica napus. FEBS Lett. 581, 1464-1474 (2007).

18. Zhang, B. H., Pan, X., Cobb, G. P. \& Anderson, T. A. Evidence that miRNAs are different from other RNAs. Cell Mol. Life Sci. 63, 246-254 (2006).

19. Yang, T., Xue, L. \& An, L. Functional diversity of miRNA in plants. Plant Sci. 172, 423-432 (2007).

20. Gupta, P. K. MicroRNAs and target mimics for crop improvement. Curr. Sci. 108, 1624-1633 (2015).

21. Zhang, B. H., Pan, X. P., Wang, Q. L., Cobb, G. P. \& Anderson, T. A. Identification and characterization of new plant microRNAs using EST analysis. Cell Res. 15, 336-360 (2005).

22. Zhang, B. H., Pan, X., Cobb, G. P. \& Anderson, T. A. Plant microRNA: a small regulatory molecule with big impact. Dev. Biol. 289, 3-16 (2006).

23. Jones, G. S., Grocock, R. J., Van Dongen, S., Bateman, A. \& Enright, A. J. MiRBase: microRNA sequences, targets and gene nomenclature. Nucl. Acids Res. 34, D140-D144 (2006).

24. Li, W. \& Godzik, A. Cd-hit: a fast program for clustering and comparing large sets of protein or nucleotide sequences. Bioinformatics 22, 1658-1659 (2006).

25. Altschul, S. F., Gish, W., Miller, W., Myers, E. W. \& Lipman, D. J. Basic local alignment search tool. J. Mol. Biol. 215, 403-410 (1990).

26. Zuker, M. Mfold web server for nucleic acid folding and hybridization prediction. Nucl. Acids Res. 31, 3406-3415 (2003).

27. Dai, X. \& Zhao, P. X. psRNATarget: a plant small RNA target analysis server. Nucl. Acids Res. 39, W155-W159 (2011).

28. Darzentas, N. Circoletto visualizing sequence similarity with Circos. Bioinformatics 26, 20 (2010).

29. Krzywinski, M. et al. Circos: an information aesthetic for comparative genomics. Genome Res. 19, 1639-1645 (2009).

30. Kumar, S., Stecher, G. \& Tamura, K. MEGA7: Molecular Evolutionary Genetics Analysis version 7.0 for bigger datasets. Mol. Biol. Evol. 33, 1870-1874 (2016)

31. Kong, L. et al. CPC, assess the protein coding potential of transcripts using sequence features and support vector machine. Nucl. Acids Res. 35, 345-W349 (2007).

32. Yi, X., Zhang, Z., Ling, Y., Xu, W. \& Su, Z. PNRD: a plant non-coding RNA database. Nucl. Acids Res. 43, D982-D989 (2015).

33. Singh, J. \& Nagaraju, J. In silico prediction and characterization of microRNAs from red flour beetle (Tribolium castaneum). Insect Mol. Biol. 17, 427-436 (2008).

34. Zhang, B. H., Pan, X. P., Cox, S. B., Cobb, G. P. \& Anderson, T. A. Evidence that miRNAs are different from other RNAs. Cell. Mol. Life Sci. 63, 246-254 (2006).

35. Wang, J., Hou, X. \& Yang, X. Identification of conserved microRNAs and their targets in Chinese cabbage (Brassica rapa subsp. pekinensis). Genome. 52, 1029-1040 (2011).

36. Ambros, V., Bartel, B. \& Bartel, D. P. A uniform system for microRNA annotation. RNA 9, 277-279 (2003).

37. Dereeper, V. et al. Phylogeny. Fr: robust phylogenetic analysis for the non-specialist. Nucl. Acids Res. 36, W465-W469 (2008).

38. Edgar, R. C. MUSCLE: multiple sequence alignment with high accuracy and high throughput. Nucl. Acids Res. 32, 1792-1797 (2004).

39. Castresana, J. Selection of conserved blocks from multiple alignments for their use in phylogenetic analysis. Mol. Biol. Evol. 17, $540-552(2000)$.

40. Guindon, S. \& Gascuel, O. A simple, fast, and accurate algorithm to estimate large phylogenies by maximum likelihood. Syst. Biol. 52, 696-704 (2003).

41. Chevenet, F., Brun, C., Banuls, A. L., Jacq, B. \& Chisten, R. TreeDyn: towards dynamic graphics and annotations for analyses of trees. BMC Bioinform. 7, 439 (2006).

42. Lohse, M. et al. Mercator: a fast and simple web server for genome scale functional annotation of plant sequence data. Plant Cell Environ. 37, 1250-1258 (2014).

43. Tian, T. et al. agriGO v2.0: a GO analysis toolkit for the agricultural community, 2017 update. Nucl. Acids Res. 45, W122-W129 (2017).

44. Conesa, A. \& Götz, S. Blast2GO: A comprehensive suite for functional analysis in plant genomics. Int. J. Plant Genomics. https:// doi.org/10.1155/2008/619832 (2008). 
45. Bonnet, E., Wuyts, J., Rouze, P. \& Van de Peer, Y. Detection of 91 potential conserved plant microRNAs in Arabidopsis thaliana and Oryza sativaidentifies important target genes. Proc. Natl. Acad. Sci. USA 101, 11511-11516 (2004).

46. Bonnet, E., Wuyts, J., Rouze, P. \& Van de Peer, Y. Evidence that microRNA precursors, unlike other non-coding RNAs, have lower folding free energies than random sequences. Bioinformatics 20, 2911-2917 (2004).

47. Yin, V. P. et al. Fgf dependent depletion of microRNA-133 promotes appendage regeneration in zebrafish. Genes Dev. 22, 728-733 (2008).

48. Sunkar, R., Li, Y. F. \& Jagadeeswaran, G. Functions of microRNAs in plant stress responses. Trends Plant Sci. 17, 196-203 (2012).

49. Zhang, B. MicroRNA: a new target for improving plant tolerance to abiotic stress. J. Exp. Bot. 66, 1749-1761 (2015).

50. Franco, N. B. et al. The micro-RNA172c-APETALA2-1 node as a key regulator of the common bean-rhizobium etli Nitrogen fixation symbiosis. Plant Physiol. 168, 273-291 (2015).

51. Kim, V. N. MicroRNA biogenesis: coordinated cropping and dicing. Nat. Rev. Mol. Cell Biol. 6, 376-385 (2005).

52. Chen, X. MicroRNA biogenesis and function in plants. FEBS Lett. 579, 5923-5931 (2005).

53. Kang, K. et al. A novel real-time PCR assay of microRNAs using S-Poly (T), a specific oligo (dT) reverse transcription primer with excellent sensitivity and specificity. PLoS ONE 7(11), e48536 (2012).

54. Zhang, H. et al. miRNA-mRNA integrated analysis reveals roles for miRNAs in a typical halophyte, Reaumuria soongorica, during seed germination under salt stress. Plants 9(3), 351 (2020).

55. Yasin, J.K. High density SSR and SNP saturated physical maps of Vigna radiata. https://legumeinfo.org/genomes/gbrowse/Vr1.0 (2018).

56. Singh, S. et al. A $62 \mathrm{~K}$ genic-SNP chip array for genetic studies and breeding applications in pigeonpea (Cajanus cajan L. Millsp.). Sci. Rep. 10, 4960. https://doi.org/10.1038/s41598-020-61889-0 (2020).

57. Rogers, K. \& Chen, X. Biogenesis, turnover, and mode of action of plant microRNAs. Plant Cell. 25, 2383-2399 (2013).

58. Dezulian, T., Palatnik, J. F., Huson, D. \& Weigel, D. Conservation and divergence of microRNA families in plants. Genome Biol. 6 , $1(2005)$.

59. Dezulian, T., Schaefer, M., Wiese, R., Weigel, D. \& Huson, D. H. CrossLink: visualization and exploration of sequence relationships between (micro) RNAs. Nucl. Acids Res. 34, W400-W404 (2006).

60. Weber, M. J. New human and mouse microRNA genes found by homology search. FEBS J. 272, 59-73 (2005).

61. Singh, N., Srivastava, S. \& Sharma, A. Identification and analysis of miRNAs and their targets in ginger using bioinformatics approach. Gene 575, 570-576 (2016).

62. Panda, D. et al. Computational identification and characterization of conserved miRNAs and their target genes in garlic (Allium sativum L.) expressed sequence tags. Gene. 537, 333-342 (2014).

63. Akter, M. M. et al. Computational identification of miRNA and targets from expressed sequence tags of coffee (Coffea arabica). Saudi J. Biol. Sci. 21, 3-12 (2014).

64. Das, A. \& Mondal, T. K. Computational identification of conserved microRNAs and their targets in tea (Camellia sinensis). Am. J. Plant Sci. 1, 77 (2010).

65. Zhang, B. H., Pan, X. P., Cox, S. B., Cobb, G. P. \& Anderson, T. A. Conservation and divergence of plant microRNA genes. Plant J. 46, 243-259 (2006).

66. Wang, L., Liu, H., Li, D. \& Chen, H. Identification and characterization of maize microRNAs involved in the very early stage of seed germination. BMC Genomics. 12, 154 (2011).

67. Stark, K. L. et al. Altered brain microRNA biogenesis contributes to phenotypic deficits in a 22q11-deletion mouse model. Nat. Genet. 40, 751-760 (2008).

68. Xie, Y. F., Shu, R., Jiang, S. Y., Liu, D. L. \& Zhang, X. L. Comparison of microRNA profiles of human periodontal diseased and healthy gingival tissues. Int. J. Oral Sci. 3, 125-134 (2011).

69. Frazier, T. P., Xie, F., Freistaedter, A., Burklew, C. E. \& Zhang, B. Identification and characterization of microRNAs and their target genes in tobacco (Nicotiana tabacum). Planta 232, 1289-1308 (2010).

70. Jha, A. \& Shankar, R. Employing machine learning for reliable miRNA target identification in plants. BMC Genomics 12, 1 (2011).

71. Ding, J., Li, D., Ohler, U., Guan, J. \& Zhou, S. Genome-wide search for miRNA-target interactions in Arabidopsis thaliana with an integrated approach. BMC Genomics 13, 1 (2012).

72. Kamthan, A., Chaudhuri, A., Kamthan, M. \& Datta, A. Small RNAs in plants: recent development and application for crop improvement. Front. Plant Sci. 6, 208 (2015).

73. Li, C. \& Zhang, B. MicroRNAs in control of plant development. J. Cell. Physiol. 231, 303-313 (2016).

74. Catalano, D., Pignone, D., Sonnante, G. \& Finetti-Sialer, M. M. In-silico and in-vivo analyses of EST databases unveil conserved miRNAs from Carthamus tinctorius and Cynara cardunculus. BMC Bioinform. 13(S4), S12 (2012).

75. Rhoades, M. W. J. \& Bartel, D. P. Computational identification of plant microRNAs and their targets, including a stress- induced miRNA. Mol. Cell. 14, 787-799 (2004).

76. Bhardwaj, S., Singh, A. \& Singh, P. MicroRNA-based cancer therapeutics: big hope from small RNAs. Mol. Cell. Pharmacol. 2, 213-219 (2010).

77. Zhai, J. et al. MicroRNAs as master regulators of the plant NB-LRR defense gene family via the production of phased, trans-acting siRNAs. Genes Dev. 25, 2540-2553 (2011).

78. Xia, R., Zhu, H., An, Y. Q., Beers, E. P. \& Liu, Z. Apple miRNAs and tasiRNAs with novel regulatory networks. Genome Biol. 13, 1 (2012).

79. Adai, A. et al. Computational prediction of miRNAs in Arabidopsis thaliana. Genome Res. 15, 78-91 (2005).

80. Zhang, L. et al. A genome-wide characterization of microRNA genes in maize. PLoS Genet. 5, e1000716 (2009).

81. Joshi, T. et al. Prediction of novel miRNAs and associated target genes in Glycine max. BMC Bioinform. 11, S14 (2010).

82. Szklarczyk, D. et al. STRING v11: protein-protein association networks with increased coverage, supporting functional discovery in genome-wide experimental datasets. Nucl. Acids Res. 47(D1), D607-D613 (2019).

83. Xu, L. et al. OrthoVenn2: a web server for whole-genome comparison and annotation of orthologous clusters across multiple species. Nucleic Acids Res. 47(W1), W52-W58 (2019).

84. Babicki, S. et al. Heatmapper: web-enabled heat mapping for all. Nucl. Acids Res. 44(W1), W147-W153 (2016).

\section{Acknowledgements}

We thank Director, Indian Council of Agricultural Research -National Bureau of Plant Genetic Resources and Officer-In-Charge, Division of Genomic Resources for providing funds (PGR/DFP-BUR-DEL-01.01) and facilities to conduct this research. The authors extend their appreciation to King Saud University, Researchers Supportig Project (RSP-2020/118).

\section{Author contributions}

Y.J.K. the corresponding author; Y.J.K., A.P. and V.C. developed the concept, planned, coordinated and executed the research. Y.J.K., B.K.M. carried out research. S.H.W. and Y.J.K. and V.C. drafted the manuscript; Y.J.K. conducted pot experiments and wet lab experiments; M.A.P. performed quantitative expression of miRs; N.V., 
S.H.W., E.H.O., E.D.O. and P.S.P. edited the manuscript. Y.J.K. modified the manuscript. All authors read, approved and accepted the manuscript submission.

\section{Funding}

This work was supported by the Indian Council of Agricultural Research -National Bureau of Plant Genetic Resources [PGR/DFP-BUR-DEL-01.01] and funded by the King Saud University through Researchers Supporting Project number (RSP-2020/118).

\section{Competing interests}

The authors declare no competing interests.

\section{Additional information}

Supplementary information is available for this paper at https://doi.org/10.1038/s41598-020-73140-x.

Correspondence and requests for materials should be addressed to J.K.Y.

Reprints and permissions information is available at www.nature.com/reprints.

Publisher's note Springer Nature remains neutral with regard to jurisdictional claims in published maps and institutional affiliations.

Open Access This article is licensed under a Creative Commons Attribution 4.0 International License, which permits use, sharing, adaptation, distribution and reproduction in any medium or format, as long as you give appropriate credit to the original author(s) and the source, provide a link to the Creative Commons licence, and indicate if changes were made. The images or other third party material in this article are included in the article's Creative Commons licence, unless indicated otherwise in a credit line to the material. If material is not included in the article's Creative Commons licence and your intended use is not permitted by statutory regulation or exceeds the permitted use, you will need to obtain permission directly from the copyright holder. To view a copy of this licence, visit http://creativecommons.org/licenses/by/4.0/.

(C) The Author(s) 2020 